\title{
The Rule of Suppressed Charge Transfer between Heteroaromatic Compounds and Electrophiles
}

\author{
Viktorija Gineityte \\ Institute of Theoretical Physics and Astronomy, Vilnius University, Gostauto 12, LT-01108 Vilnius, Lithuania \\ (E-mail:Viktorija.Gineityte@tfai.vu.lt) \\ RECEIVED NOVEMBER 4, 2010; REVISED DECEMBER 11, 2012; ACCEPTED FEBRUARY 20, 2013
}

\begin{abstract}
Replacement of a carbon atom of an aromatic compound by a more electronegative heteroatom gives rise to suppression of reactivities of the remaining carbon atoms in electrophilic substitution $\left(\mathrm{S}_{\mathrm{E}} 2\right)$ reactions. To prove this experiment-based rule, the reactivities are related to the relevant extents of the intermolecular charge transfer. General algebraic expressions are derived and analyzed for these extents for various directions of the attack of electrophile. The reactant is considered as a perturbed alternant hydrocarbon, the perturbation coinciding with the alteration in the Coulomb parameter of the carbon atom undergoing the replacement, whereas the reagent is represented explicitly by an external vacant orbital. Changes in the intermolecular charge transfer due to introduction of heteroatom are shown to consist of two components, viz. of an initial-population-dependent component contributing increments of alternating signs for shifting positions of electrophile along the hydrocarbon chain and of an additional negative contribution originating from the increased interval between diagonal Hamiltonian matrix elements for orbitals participating in the charge transfer. For soft electrophiles, the negative contribution is shown to predominate over the alternating one. This implies the charge transfer between heteroaromatic reactants and electrophiles to be suppressed $v s$. the relevant values for hydrocarbons-containing system. (doi: $10.5562 / \mathrm{cca} 1794)$
\end{abstract}

Keywords: alternant conjugated hydrocarbons, the Hückel model, chemical reactivity, electrophilic aromatic substitution, soft electrophiles

\section{INTRODUCTION}

Relative reactivities of related chemical compounds towards a certain reagent usually exhibit more or less regular behaviour. This allows us to formulate definite rules and/or laws and to use them instead of extended collections of separate facts. Generalizations of just this type form the basis of chemistry as an exact science.

The organic chemistry is no exception in this respect. Comparative studies of electrophilic substitution $\left(\mathrm{S}_{\mathrm{E}} 2\right)$ processes of aromatic and heteroaromatic compounds ${ }^{1-6}$ may be taken here as an excellent example. Indeed, the heteroatom-containing reactants usually are characterized by much slower rates of substitution $v s$. their parent hydrocarbons. ${ }^{5-9}$ This especially refers to aromatic heterocycles with six-membered rings (e.g. pyridine, quinoline, isoquinoline, acridine, various phenylpyridines, etc.). ${ }^{5,7,8}$ For instance, even the most reactive meta positions of pyridine are considerably less reactive $v s$. individual atoms of benzene, ${ }^{5,8}$ Analogous suppressions of reactivities are peculiar also to de- rivatives of aromatic hydrocarbons with electronaccepting substituents, such as the $\mathrm{NO}_{2}, \mathrm{CN}, \mathrm{COH}$ and related groups. ${ }^{2,10}$

The regular nature of the above-overviewed facts allows us to assume them to obey the following rule, viz. replacement of a carbon atom of an aromatic hydrocarbon by a more electronegative heteroatom gives rise to suppression of relative reactivities of all the remaining carbon atoms. To support this hypothesis, we will look for a quantum-chemical analogue of the same rule. Apart from corroborating the law-like character of experimental facts, such an analogue (if derived algebraically and/or proven rigorously) would be able to provide us with their common accounting, as well as to reveal additional conditions underlying the rule (if any).

To achieve these ends, we have to choose an appropriate way of quantum-chemical description of $\mathrm{S}_{\mathrm{E}} 2$ reactions. The relevant literature offers us a large selection of approaches of various degrees of sophistication, starting with the simplest models (e.g. relating the relative reactivities to respective populations 
of $2 \mathrm{p}_{z}$ AOs of isolated reactants following from the Hückel (HMO) theory ${ }^{5,6,11,12}$ ) and ending with qualitative constructions ${ }^{13}$ and modern computations $^{14-21}$ of potential energy surfaces. Numerous applications of the popular paradigm of charge- and frontier-orbital-controlled reactions ( $c f$. the so-called HOMO/LUMO concept $)^{10,22-30}$ take an intermediate place in between the above-mentioned two extremes. The same refers also to more elaborated reactivity descriptors $^{31-39}$ including those formulated in the framework of the density functional theory (DFT). ${ }^{35-39}$ It deserves adding here that both the original HOMO/LUMO concept ${ }^{22-27}$ and its analogues in terms of the DFT $^{35,37,39-44}$ (including the Fukui function ${ }^{40-44}$ ) are based on consideration of the charge transfer between the reactant and the reagent (the frontierorbital-controlled reactions virtually are charge-transfercontrolled ones ${ }^{35}$ ). From analysis of these contributions an immediate observation follows that the more sophisticated approach is applied, the less general results are obtained. As for instance, populations of $2 \mathrm{p}_{z}$ AOs of heteroaromatic molecules are governed by the rule of the alternating polarity ${ }^{45-47}$ in the simple Hückel theory that takes a theorem-like form and has been proven rigorously for an extended class of compounds (see below). For comparison, the DFT along with the HOMO/LUMO concept yields only a verbal explanation of suppressed reactivities of particular nitrogencontaining heterocycles. ${ }^{8}$ On the other hand, the rich experience of quantum chemistry shows that simple approaches sometimes yield wrong predictions. Thus, we need an approach emboding a fortunate combination of both adequacy and generality.

Let us dwell first on the above-described simplest approach based on the Hückel theory. Aromatic heterocycles and other hetero-conjugated compounds are usually regarded in this theory as perturbed hydrocarbons, the perturbation consisting of a certain alteration in the Coulomb parameter $(\alpha)$ of the carbon atom undergoing the replacement. The majority of the relevant parent systems, in turn, belong to the so-called alternant hydrocarbons $(\mathrm{AHs})^{5,6,11,12,45-48}$ that are representable by bipartite graphs of their C-skeletons. $^{49-51}$ Just this implies the validity of the rule of alternating polarity ${ }^{45-47}$ being interpretable in graphtheoretical terms too. ${ }^{46}$ As a result, populations of AOs of the heteroatom-containing subset of any derivative are predicted to be increased, whilst those of the remaining subset are expected to be reduced $v s$. uniform occupation numbers of all AOs of an $\mathrm{AH}$ equal to unity $^{49,50}$ (The well-known partition of the basis set of $2 \mathrm{p}_{z}$ AOs of carbon atoms of an $\mathrm{AH}$ into two subsets ${ }^{48-51}$ is invoked here). On this basis, enhanced relative reactivities are anticipated for all positions of the derivative represented by AOs of the first subset in direct contradiction to the above-discussed experimental facts. This failure was noticed a long time ago ${ }^{5,11}$ and it was entirely ascribed to the seemingly oversimplified nature of the very Hückel model. In this connection, numerous attempts ${ }^{5,11}$ were undertaken to 'improve' charge redistributions in hetero-conjugated molecules by turning to the Pariser-Parr-Pople (PPP) method and some of these attempts proved to be successful. The relevant calculations performed later by means of $a b$ initio methods, however, did not corroborate these 'improvements'. Quite the reverse, both total charges on atoms and populations of $2 \mathrm{p}_{z}$ AOs exhibit alternation over the hydrocarbon chain in pyridine ${ }^{52-55}$ that acquired also an experimental support. ${ }^{52}$ The results of the $\mathrm{RHF} / / 6-31 \mathrm{G}^{* *}$ method $^{55}$ are especially noteworthy here, as the absolute value of the negative charge of the meta-positioned carbon atom of pyridine exceeds considerably that of benzene in contrast to observed relative reactivities. ${ }^{5,8}$ It deserves also adding here that the very rule of the polarity alternation also did not lose its significance and is successfully applied in the computer-assisted synthesis planning. ${ }^{56-58}$

We may assume, therefore, that relating the relative reactivities to populations of AOs of the isolated reactant calls forth the above-discussed failure rather than deficiences of the HMO theory. In this connection, we will invoke the actual extents of charge transfer between the reactant and the reagent as the principal criterion of relative reactivity in the present study. To this end, both the approaching electrophile and its interaction with the reactant will be considered explicitly. So far as the reactant itself is concerned, the above-described classical model of an $\mathrm{AH}$ and of its perturbed analogue will be preserved. That is why we expect to be able to obtain a new common rule like that of the alternating polarity.

The actual extents of charge transfer between participants of $\mathrm{S}_{\mathrm{E}} 2$ processes evidently depend on the relevant intermolecular resonance parameters. To eliminate this superfluous dependence, we will define the so-called charge transfer (CT) abilities of the system for different routes of the reaction. Our principal aim then consists in derivation of general algebraic expressions for these abilities and, especially, for their alterations when passing from an $\mathrm{AH}$ to its perturbed analogue, the latter conveniently referred to as the perturbed $\mathrm{AH}(\mathrm{PAH}){ }^{47}$ Thereupon, we are about to establish the signs of these alterations for various positions of the reagent. Revealing of the relation between the CT ability of the system for the attack of electrophile upon a certain AO of the reactant and the initial population of the same $\mathrm{AO}$ also is under interest in the present context.

The common Hückel type Hamiltonian matrix of AHs usually is initially represented in the basis of $2 p_{z}$ 
AOs of carbon atoms. ${ }^{45,47-50}$ Recently, this matrix was shown to be transformable into a block-diagonal form. ${ }^{59}$ This achievement, in turn, allowed us to apply the socalled non-commutative Rayleigh-Schrödinger perturbation theory (NCRSPT) $)^{60-62}$ to analyze charge redistributions in AHs due to various perturbations. ${ }^{47,63,64}$ Alteration in a certain Coulomb parameter ( $\alpha$ ) has been considered as the simplest example and the rule of the alternating polarity has been rederived. ${ }^{47}$ An external electron-donating (accepting) subsystem has been attached later to an $\mathrm{AH}$ and the consequent intersubsystem charge transfer has been studied. ${ }^{64}$ The resonance parameter $(v)$ describing the interaction between this additional subsystem and the relevant $\mathrm{AO}$ of the parent $\mathrm{AH}$ served as perturbation in the latter case. An approaching electrophile also may be modeled similarly. To study the alterations in the intersubsystem charge transfer when passing from an $\mathrm{AH}$ to its derivative, we have now to combine the perturbations $\alpha$ and $v$ and to extend the previous methodo$\operatorname{logy} y^{47,59,63,64}$ for analysis of non-additive effects of these perturbations (i.e. of consequences of their interplay).

The paper starts with a brief description of the very mathematical problem and of its solution. Thereupon, we derive the expressions for the CT abilities. The final sections are devoted to analysis of the results and to their illustration.

\section{METHODOLOGY}

As already mentioned, we will consider an $\mathrm{AH}$ and its derivative under attack of an external reagent (electrophile). Moreover, the latter will be assumed to attack particular carbon atoms in accordance with the classical mechanism of $\mathrm{S}_{\mathrm{E}} 2$ reactions. The first step of the study consists in constructing the relevant model Hamiltonian matrices.

Let us start with the zero order matrix. In the simple Hückel model, the $2 n$-dimensional basis set $\{\chi\}$ of any even $\mathrm{AH}$ consisting of $2 \mathrm{p}_{z}$ AOs of carbon atoms is known to be divisible into two $n$-dimensional subsets $\left\{\chi^{*}\right\}$ and $\left\{\chi^{\circ}\right\}$ so that the intrasubset resonance parameters take zero values. ${ }^{47-50,59}$ Given that the Coulomb parameters $\left(\alpha_{C}\right)$ are additionally assumed to be uniform and the equality $\alpha_{C}=0$ is accepted for convenience, the model Hamiltonian matrices of AHs acquire a common form containing non-zero submatrices (blocks) in its off-diagonal positions only as exhibited below in Eq. (1). The mean value of resonance parameters between neighboring pairs of AOs of the $\mathrm{AH}(\beta)$ will serve as a (negative) energy unit in our study, i.e. the equality $\beta=1$ will be accepted.

Let the reagent (electrophile) to be represented by a single vacant orbital as usual in accordance with the well-known electron-deficient nature of this spe- cies. ${ }^{1,3-6,10,12,29}$ This implies the electrophile to contribute no additional electrons to the initial system of $2 n$ electrons of the reactant (substrate). The abovespecified additional orbital will be denoted by $\varphi_{(-) e}$ and characterized by the energy parameter $-\mu$, where $\mu>0$ and the minus sign is introduced for convenience (the meaning of the subscript $(-)$ is clarified below). The relative order of orbitals of the total $2 n+1$ dimensional basis set will be chosen to coincide with $\left\{\chi^{*}\right\},\left\{\chi^{0}\right\}$ and $\varphi_{(-) e}$ The zero order Hamiltonian matrix of our system $\left(\boldsymbol{H}_{(0)}\right)$ takes then the form

$$
\boldsymbol{H}_{(0)}=\left|\begin{array}{ccc}
\mathbf{0} & \boldsymbol{B} & \mathbf{0} \\
\boldsymbol{B}^{+} & \mathbf{0} & \mathbf{0} \\
\mathbf{0} & \mathbf{0} & -\mu
\end{array}\right|,
$$

where submatrices $\boldsymbol{B}$ and $\boldsymbol{B}^{+}$contain the intersubset resonance parameters of the parent $\mathrm{AH}$ [The superscript + here and below represents the Hermitian-conjugate (transposed) matrix]. Non-zero elements $B_{i j}$ coincide with parameters referring to the neighboring pairs of AOs. Under an additional condition of uniform values of the latter, the submatrices $\boldsymbol{B}$ and $\boldsymbol{B}^{+}$coincide with respective blocks of adjacency matrices of graphs describing the $\mathrm{C}$-skeletons of the parent $\mathrm{AH} .{ }^{49-51}$

Let us assume now that the 1st carbon atom represented by the $\mathrm{AO} \chi_{1}^{*}$ is replaced by a slightly more electronegative heteroatom. As a result, an alteration in the relevant Coulomb parameter arises. Let this intramolecular perturbation to be denoted by $\alpha$. The resonance parameter of the intermolecular type describing the interaction between the orbital $\varphi_{(-) e}$ and the $\mathrm{AO}$ of the carbon atom under attack of electrophile will be designated by $v$ and supposed to take a sufficiently small absolute value as compared to our energy unit. Thus, the parameter $v$ also will be included into the first order Hamiltonian matrix. So far as the direction of an electrophilic attack is concerned, two cases may be distinguished, namely an attack upon an orbital $\chi_{k}^{*}$ of the first subset $\left\{\chi^{*}\right\}$ and that upon an AO $\chi_{k}^{\mathrm{o}}$ of the second subset $\left\{\chi^{\mathrm{o}}\right\}$. Two alternative perturbation matrices $\left(\boldsymbol{H}_{(1)}^{*}\right.$ and $\left.\boldsymbol{H}_{(1)}^{\circ}\right)$ may be accordingly defined. These are

$$
\boldsymbol{H}_{(1)}^{*}=\left|\begin{array}{ccc}
\boldsymbol{A}_{(1)} & \mathbf{0} & \boldsymbol{a} \\
\mathbf{0} & \mathbf{0} & \mathbf{0} \\
\boldsymbol{a}^{+} & \mathbf{0} & 0
\end{array}\right|, \quad \boldsymbol{H}_{(1)}^{\mathrm{o}}=\left|\begin{array}{ccc}
\boldsymbol{A}_{(1)} & \mathbf{0} & \mathbf{0} \\
\mathbf{0} & \mathbf{0} & \boldsymbol{a} \\
\mathbf{0} & \boldsymbol{a}^{+} & 0
\end{array}\right|,
$$

where the $n \times n$-dimensional submatrix $\boldsymbol{A}_{(1)}$ involves a single non-zero element $A_{(1) 11}=\alpha$ and $\boldsymbol{a}$ is a columnmatrix containing a non-zero element $a_{k}=v$. It deserves an additional emphasizing that the total number of electrons coincides with that of $2 p_{z}$ AOs of the reactant $(2 n)$ in the perturbed systems too. 
Both the Hückel type Hamiltonian matrix of $\mathrm{Ahs}^{59}$ and the matrix $\boldsymbol{H}_{(0)}$ of Eq. (1) ${ }^{64}$ were shown to be transformable into block-diagonal forms using a certain unitary matrix $(\boldsymbol{C})$ specified below. The same transformation may be subsequently applied to first order matrices $\boldsymbol{H}_{(1)}^{*}$ and $\boldsymbol{H}_{(1)}^{\mathrm{o}}$. Let the new total Hamiltonian matrices to be designated by $\tilde{\boldsymbol{H}}^{*}$ and $\tilde{\boldsymbol{H}}^{\circ}$ and note both of them to contain the same blockdiagonal zero order member $\left(\tilde{\boldsymbol{H}}_{(0)}\right)$. For any Hamiltonian matrix of this type, the relevant representation of the one-electron density matrix (DM) $\tilde{\boldsymbol{P}}$ has been derived by means of a direct solution of the commutation equation in the form of power series. ${ }^{65-68}$ Thus, we may now apply the same series to construct the DMs $\tilde{\boldsymbol{P}}^{*}$ and $\tilde{\boldsymbol{P}}^{\mathrm{o}}$ referring to Hamiltonian matrices $\tilde{\boldsymbol{H}}^{*}$ and $\tilde{\boldsymbol{H}}^{\circ}$, respectively. Thereupon, the results may be retransformed into the basis of AOs $\{\chi\}$ again (if necessary). Just the above-outlined procedure underlies our methodology. Let us consider now the relevant expressions.

Let us introduce the following $n \times n$-dimensional Hermitian (symmetric) matrices ${ }^{47,48,59,63,64}$

$$
\boldsymbol{R}=\left(\boldsymbol{B} \boldsymbol{B}^{+}\right)^{-1 / 2}, \quad \boldsymbol{Q}=\left(\boldsymbol{B}^{+} \boldsymbol{B}\right)^{-1 / 2},
$$

where the positive-definite square roots are assumed to be chosen. Two alternative representations of the transformation matrix $\boldsymbol{C}$ may be obtained. ${ }^{59}$ The socalled $\boldsymbol{Q}$-representation takes the form

$$
C=\frac{1}{\sqrt{2}}\left|\begin{array}{ccc}
\boldsymbol{I} & \boldsymbol{B} \boldsymbol{Q} & \mathbf{0} \\
\boldsymbol{Q B} \boldsymbol{B}^{+} & -\boldsymbol{I} & \mathbf{0} \\
\mathbf{0} & 0 & \sqrt{2}
\end{array}\right|,
$$

where $\boldsymbol{I}$ stands for the unit matrix. The $\boldsymbol{R}$ representation of the same matrix results from Eq. (4) after replacing the product $\boldsymbol{B} \boldsymbol{Q}$ by $\boldsymbol{R} \boldsymbol{B}$ in accordance with their coincidence, viz.

$$
R B=B Q
$$

Moreover, the unitarity condition ${ }^{59}$ for the matrix $\boldsymbol{C}$ yields the relations

$$
R B B^{+} R=Q B^{+} B Q=B Q Q B^{+}=B^{+} R R B=I .
$$

The transformed zero order Hamiltonian matrix takes then the form

$$
\tilde{\boldsymbol{H}}_{(0)}=\boldsymbol{C}^{+} \boldsymbol{H}_{(0)} \boldsymbol{C}=\left|\begin{array}{ccc}
\boldsymbol{E}_{(+)} & \mathbf{0} & \mathbf{0} \\
\mathbf{0} & -\boldsymbol{E}_{(-)} & \mathbf{0} \\
\mathbf{0} & \mathbf{0} & -\mu
\end{array}\right|,
$$

where $\boldsymbol{E}_{(+)}$and $\boldsymbol{E}_{(-)}$are the following $n \times n$-dimensional submatrices

$$
\boldsymbol{E}_{(+)}=\boldsymbol{R}^{-1}=\left(\boldsymbol{B} \boldsymbol{B}^{+}\right)^{1 / 2}, \quad \boldsymbol{E}_{(-)}=\boldsymbol{Q}^{-1}=\left(\boldsymbol{B}^{+} \boldsymbol{B}\right)^{1 / 2}(8)
$$

coinciding with the so-called eigenblocks of the Hamiltonian matrix of the parent AHs. ${ }^{59}$ For transformed first order Hamiltonian matrices, we accordingly obtain

$$
\begin{aligned}
\tilde{\boldsymbol{H}}_{(1)}^{*} & =\boldsymbol{C}^{+} \boldsymbol{H}_{(1)}^{*} \boldsymbol{C}= \\
& =\frac{1}{2}\left|\begin{array}{ccc}
\boldsymbol{A}_{(1)} & \boldsymbol{A}_{(1)} \boldsymbol{B} \boldsymbol{Q} & \sqrt{2} \boldsymbol{a} \\
\boldsymbol{Q} \boldsymbol{B}^{+} \boldsymbol{A}_{(1)} & \boldsymbol{Q} \boldsymbol{B}^{+} \boldsymbol{A}_{(1)} \boldsymbol{B} \boldsymbol{Q} & \sqrt{2} \boldsymbol{Q} \boldsymbol{B}^{+} \boldsymbol{a} \\
\sqrt{2} \boldsymbol{a}^{+} & \sqrt{2} \boldsymbol{a}^{+} \boldsymbol{B} \boldsymbol{Q} & 0
\end{array}\right|, \\
\tilde{\boldsymbol{H}}_{(1)}^{\mathrm{o}} & =\boldsymbol{C}^{+} \boldsymbol{H}_{(1)}^{\mathrm{o}} \boldsymbol{C}= \\
& =\frac{1}{2}\left|\begin{array}{ccc}
\boldsymbol{A}_{(1)} & \boldsymbol{A}_{(1)} \boldsymbol{B} \boldsymbol{Q} & \sqrt{2} \boldsymbol{B} \boldsymbol{Q} \boldsymbol{a} \\
\boldsymbol{Q} \boldsymbol{B}^{+} \boldsymbol{A}_{(1)} & \boldsymbol{Q} \boldsymbol{B}^{+} \boldsymbol{A}_{(1)} \boldsymbol{B} \boldsymbol{Q} & -\sqrt{2} \boldsymbol{a} \\
\sqrt{2} \boldsymbol{a}^{+} \boldsymbol{Q} \boldsymbol{B}^{+} & -\sqrt{2} \boldsymbol{a}^{+} & 0
\end{array}\right| .
\end{aligned}
$$

It is noteworthy here that the transformation of Eq. (7) deals with entire blocks of matrices concerned instead of matrix elements as usual. ${ }^{60-62}$ That is why the procedure does not affect the dimensions of these blocks. Furthermore, the above-described transformation represents passing to the basis of non-canonical molecular orbitals (NCMOs) of the parent $\mathrm{AH}^{59}$ Explicit expressions for these orbitals follow directly from Eq. (4) and take the form of linear combinations of $2 \mathrm{p}_{z}$ AOs $\{\chi\}$. Distinctive features of NCMOs of AHs have been analyzed in a detail ${ }^{59}$. An important point in our context is that NCMOs of even AHs may be unambiguosly classified into $n$ occupied and $n$ vacant orbitals on the basis of their occupation numbers (populations) coinciding either with 2 or 0 as discussed below. Let the $n$-dimensional subsets of occupied and vacant NCMOs of our AH to be denoted by $\left\{\Psi_{(+)}\right\}$and $\left\{\Psi_{(-)}\right\}$, where the subscripts $(+)$and $(-)$indicate their occupations. The total $(2 n+1)$-dimensional basis set then contains the above-defined subsets and the vacant orbital $\varphi_{(-) e}$. Accordingly, submatrices of first order matrices $\tilde{\boldsymbol{H}}_{(1)}^{*}$ and $\tilde{\boldsymbol{H}}_{(1)}^{o}$ represent interactions of these basis orbitals due to perturbations. In particular, the intersubsystem blocks $(\sqrt{2})^{-1} \boldsymbol{a}$ and $(\sqrt{2})^{-1} \boldsymbol{Q} \boldsymbol{B}^{+} \boldsymbol{a}$, as well as $(\sqrt{2})^{-1} \boldsymbol{B} \boldsymbol{Q} \boldsymbol{a}$ and $-(\sqrt{2})^{-1} \boldsymbol{a}$ contain the interactions between NCMOs of the reactant and the orbital $\varphi_{(-) e}$. Meanwhile, the intramolecular perturbation $\alpha$ is formally present only within blocks of matrices $\tilde{\boldsymbol{H}}_{(1)}^{*}$ and $\tilde{\boldsymbol{H}}_{(1)}^{0}$ referring to the reactant. In our 
context, the submatrix (1/2) $\boldsymbol{A}_{(1)}$ is of particular interest. Indeed, the structure of this submatrix indicates that the diagonal Hamiltonian matrix element of only a single occupied NCMO is changed by $(1 / 2) \alpha$ due to introduction of the heteroatom. This exclusive NCMO is attached to the site of the heteroatom ${ }^{59}\left(\chi_{1}^{*}\right)$ and may be accordingly denoted by $\Psi_{(+) 1}$. Presence of the intersubset coupling block $\frac{1}{2} \boldsymbol{A}_{(1)} \boldsymbol{B} \boldsymbol{Q}$, in turn, implies emergence of mixing between occupied and vacant NCMOs of the parent AH. Finally, the common $\boldsymbol{A}_{(1)}{ }^{-}$ containing part of matrices $\tilde{\boldsymbol{H}}_{(1)}^{*}$ and $\tilde{\boldsymbol{H}}_{(1)}^{\mathrm{o}}$ coincides with the relevant first order Hamiltonian matrix of PAHs. ${ }^{47}$

Let us turn now to derivation of DMs $\tilde{\boldsymbol{P}}^{*}$ and $\tilde{\boldsymbol{P}}^{\text {o }}$, referring to Hamiltonian matrices $\tilde{\boldsymbol{H}}^{*}$ and $\tilde{\boldsymbol{H}}^{\text {o }}$, separate members of which are defined by Eqs. (7) and (9). As with any one-electron $\mathrm{DM}^{69}$, these matrices are generally determined by three matrix equations ${ }^{68,69}$, viz.

$$
[\tilde{\boldsymbol{H}}, \tilde{\boldsymbol{P}}]_{-}=\mathbf{0}, \quad(\tilde{\boldsymbol{P}})^{2}=2 \tilde{\boldsymbol{P}}, \quad \operatorname{Spur} \tilde{\boldsymbol{P}}=2 n,
$$

where $\tilde{\boldsymbol{H}}$ and $\tilde{\boldsymbol{P}}$ acquire the superscripts either $*$ or ${ }^{\text {o, }}$, and the notation $[\ldots, \ldots]$. indicates a commutator of matrices. Given that the Hamiltonian matrix consists of zero and first order terms (as it is the case with matrices $\tilde{\boldsymbol{H}}^{*}$ and $\tilde{\boldsymbol{H}}^{\mathrm{o}}$ ), the DM $\tilde{\boldsymbol{P}}$ may be sought in the form of power series ${ }^{65,68}$, i.e. as a sum over an order paramer $(k)$ of increments $\tilde{\boldsymbol{P}}_{(\mathrm{k})}(\mathrm{k}=0,1,2, \ldots)$. Substituting such a series into Eq.(10) followed by collecting of terms of the same order $(k)$ and by a subsequent imposing of the relevant matrix conditions within each order separately provides us with equations determining individual increments $\tilde{\boldsymbol{P}}_{(\mathrm{k})}$.

For the starting member of our series $\left(\tilde{\boldsymbol{P}}_{(0)}\right)$, the resulting three matrix equations resemble those of Eq.(10), except for the block-diagonalized zero order Hamiltonian $\tilde{\boldsymbol{H}}_{(0)}$ of Eq.(7) standing instead of the matrix $\tilde{\boldsymbol{H}}$. The solution $\tilde{\boldsymbol{P}}_{(0)}$ representing the ground state of the system ${ }^{69}$ then contains an $n$-dimensional block $2 \boldsymbol{I}$ in its first diagonal position referring to the subset $\left\{\Psi_{(+)}\right\}$and zero blocks elsewhere. This implies the NCMOs of our parent $\mathrm{AH} \Psi_{(+) i}, i=1,2 \ldots n$ and $\Psi_{(-) l}, l=1,2 \ldots n$ to be double-occupied and vacant, respectively. As a result, we may then define total subsets of occupied and vacant basis functions $\left\{\Psi_{1}\right\}$ and $\left\{\Psi_{2}\right\}$. In our case, the above-mentioned subsets contain the occupied NCMOs of the parent AH $\left(\left\{\Psi_{1}\right\} \equiv\left\{\Psi_{(+)}\right\}\right)$ and the vacant NCMOs along with the orbital of electrophile $\left(\left\{\Psi_{2}\right\} \equiv\left\{\left\{\Psi_{(-)}\right\}, \varphi_{(-) e}\right\}\right)$. [Note that these orbitals represent the unperturbed system. Occupied and vacant NCMOs of the perturbed system may be found as described in Section 3 of Ref. 65, as well as in Ref. 47.]

It deserves mentioning here that the last relation of Eq.(10) embraces systems with different total numbers of basis orbitals and of electrons in contrast to the charge conservation condition of Refs.59 and 65 (see Eqs. (30) and (6), respectively). This extension, however, does not affect the derivation ${ }^{65}$ of the subsequent members of the same series $\left(\tilde{\boldsymbol{P}}_{(k)}\right)$. As in previous studies ${ }^{65-68}$, each matrix $\tilde{\boldsymbol{P}}_{(k)}$ may be conveniently represented in terms of four submatrices (blocks) corresponding to subsets $\left\{\Psi_{1}\right\}$ and $\left\{\Psi_{2}\right\}$ and to their interaction. Let the blocks referring to separate subsets and taking the diagonal positions within corrections $\tilde{\boldsymbol{P}}_{(k)}$ to be denoted by $\tilde{\boldsymbol{P}}_{1(k)}$ and $\tilde{\boldsymbol{P}}_{2(k)}$, respectively. The intrasubset blocks $\tilde{\boldsymbol{P}}_{1}$ and $\tilde{\boldsymbol{P}}_{2}$ of the total one-electron DM $\tilde{\mathbf{P}}$ are then accordingly representable as sums over $k$ of the above-mentioned increments $\tilde{\boldsymbol{P}}_{1(k)}$ and $\tilde{\boldsymbol{P}}_{2(k)}$. Let us note immediately that just the above-specified blocks are of particular interest in our context. Indeed, their diagonal elements yield alterations in occupation numbers of respective basis orbitals due to perturbations of Eq.(9).

The most convenient way of analysis of the charge transfer between the reactant and electrophile consists in consideration of the actual population of the orbital $\varphi_{(-) e}$, resulting from the last diagonal element of the block $\tilde{\boldsymbol{P}}_{2}$. Thus, we may confine ourselves here to expressions for this block. The first and the second order members of the relevant power series $\left(\tilde{\boldsymbol{P}}_{2(1)}\right.$ and $\left.\tilde{\boldsymbol{P}}_{2(2)}\right)$ follow directly from Eqs. (29) and (37) of Ref. 65, whereas terms of higher orders may be easily obtained by a straightforward extending of the same derivation to $k=3,4$.. etc., as discussed in Refs. $66-68$, as well as 70 and 71 . The overall result is then as follows

$$
\begin{aligned}
& \tilde{\boldsymbol{P}}_{2(1)}=\mathbf{0}, \quad \tilde{\boldsymbol{P}}_{2(2)}=2 \boldsymbol{G}_{(1)}^{+} \boldsymbol{G}_{(1)}, \\
& \tilde{\boldsymbol{P}}_{2(3)}=2\left(\boldsymbol{G}_{(1)}^{+} \boldsymbol{G}_{(2)}+\boldsymbol{G}_{(2)}^{+} \boldsymbol{G}_{(1)}\right), \text { etc. }
\end{aligned}
$$

where $\boldsymbol{G}_{(k)}(k=1,2,3 \ldots)$ are the principal matrices of the NCRSPT conditioned by matrix equations of the following common form

$$
\boldsymbol{E}_{1} \boldsymbol{G}_{(k)}-\boldsymbol{G}_{(k)} \boldsymbol{E}_{2}+\boldsymbol{V}_{(k)}=\mathbf{0},
$$

(see Eqs. (27) and (35) of Ref. 65). Notations $\boldsymbol{E}_{1}$ and $\boldsymbol{E}_{2}$ stand here for zero order eigenblocks referring to subsets $\left\{\Psi_{1}\right\}$ and $\left\{\Psi_{2}\right\}$, i.e.

$$
\boldsymbol{E}_{1}=\boldsymbol{E}_{(+)}, \quad \boldsymbol{E}_{2}=\left|\begin{array}{cc}
-\boldsymbol{E}_{(-)} & \mathbf{0} \\
\mathbf{0} & -\mu
\end{array}\right|,
$$

whilst $\boldsymbol{V}_{(1)}$ and $\boldsymbol{V}_{(2)}$ are matrices of interorbital 
interactions defined below:

The first order matrix $\boldsymbol{V}_{(1)}$ coincides with the relevant total matrix of the intersubset resonance parameters. From Eq. (9), we obtain

$$
\begin{aligned}
& \boldsymbol{V}_{(1)}^{*}=\frac{1}{2}\left|\boldsymbol{A}_{(1)} \boldsymbol{B} \boldsymbol{Q} \quad \sqrt{2} \boldsymbol{a}\right|, \\
& \boldsymbol{V}_{(1)}^{\mathrm{o}}=\frac{1}{2}\left|\boldsymbol{A}_{(1)} \boldsymbol{B} \boldsymbol{Q} \quad \sqrt{2} \boldsymbol{B} Q \boldsymbol{a}\right| \text {. }
\end{aligned}
$$

The column-matrices $(\sqrt{2})^{-1} \boldsymbol{a}$ and $(\sqrt{2})^{-1} \boldsymbol{B} \boldsymbol{Q} \boldsymbol{a}$ are attached here to the common square matrix $\frac{1}{2} \boldsymbol{A}_{(1)} \boldsymbol{B} \boldsymbol{Q}$. The second order matrix $\boldsymbol{V}_{(2)}$, in turn, is constructed on the basis of the following relation (see Eq. (32) of Ref. 65)

$$
\boldsymbol{V}_{(2)}=\boldsymbol{S} \boldsymbol{G}_{(1)}-\boldsymbol{G}_{(1)} \boldsymbol{T},
$$

where $\boldsymbol{S}$ and $\boldsymbol{T}$ are first order corrections to eigenblocks coinciding with intrasubset blocks of the relevant perturbation matrix. From Eq. (9) it follows that

$$
\begin{aligned}
& \boldsymbol{S}^{*}=\boldsymbol{S}^{\mathrm{o}}=\frac{1}{2} \boldsymbol{A}_{(1)}, \\
& \boldsymbol{T}^{*}=\frac{1}{2}\left|\begin{array}{cc}
\boldsymbol{Q} \boldsymbol{B}^{+} \boldsymbol{A}_{(1)} \boldsymbol{B} \boldsymbol{Q} & \sqrt{2} \boldsymbol{Q} \boldsymbol{B}^{+} \boldsymbol{a} \\
\sqrt{2} \boldsymbol{a}^{+} \boldsymbol{B} \boldsymbol{Q} & 0
\end{array}\right|, \\
& \boldsymbol{T}^{\mathrm{o}}=\frac{1}{2}\left|\begin{array}{cc}
\boldsymbol{Q B} \boldsymbol{B}^{+} \boldsymbol{A}_{(1)} \boldsymbol{B} \boldsymbol{Q} & -\sqrt{2} \boldsymbol{a} \\
-\sqrt{2} \boldsymbol{a}^{+} & 0
\end{array}\right| .
\end{aligned}
$$

Now, no more is required as to analyze and solve the principal equation (12) under employment of particular expressions of Eqs. (13)-(16). The solution should be then substituted into Eq. (11) and the population of the orbital $\varphi_{(-) e}$ follows in the form of power series.

\section{EXPRESSIONS FOR CHARGE TRANSFER ABILITIES}

As is seen from Eq. (11), the first order member $\tilde{\boldsymbol{P}}_{2(1)}$ of the power series for the DM block $\tilde{\boldsymbol{P}}_{2}$ coincides with a zero matrix, whilst the relevant second order increment is determined by the first order matrix $\boldsymbol{G}_{(1)}$. Thus, let us start with the matrix equation of Eq. (12) for $k=1$. Since $\boldsymbol{E}_{1}$ and $\boldsymbol{E}_{2}$ are square matrices, constitution of the matrix $\boldsymbol{G}_{(1)}$ being sought is conditioned by that of $\boldsymbol{V}_{(1)}$. Thus, let us look for the matrix $\boldsymbol{G}_{(1)}$ of the following form

$$
\boldsymbol{G}_{(1)}=\left|\begin{array}{ll}
\boldsymbol{G}_{(1)}^{(\alpha)} & \boldsymbol{g}_{(1)}
\end{array}\right|,
$$

where $\boldsymbol{G}_{(1)}^{(\alpha)}$ is a square $n \times n$-dimensional submatrix and $\boldsymbol{g}_{(1)}$ is a column-matrix of the same dimension. Substitution of Eqs. (8), (13), (14) and (17) into Eq. (12) for $k=1$ shows that the latter relation may be decomposed into two independent matrix equations determining the submatrices $\boldsymbol{G}_{(1)}^{(\alpha)}$ and $\boldsymbol{g}_{(1)}$ separately. The first of these newly-derived equations contains the intersubset coupling matrix $\frac{1}{2} \boldsymbol{A}_{(1)} \boldsymbol{B} \boldsymbol{Q}$ only and depends on parameters of electrophile neither explicitly nor implicitly, viz.

$$
\boldsymbol{G}_{(1)}^{(\alpha)} \boldsymbol{Q}+\boldsymbol{R} \boldsymbol{G}_{(1)}^{(\alpha)}+\frac{1}{2} \boldsymbol{R} \boldsymbol{A}_{(1)} \boldsymbol{B} \boldsymbol{Q}^{2}=\mathbf{0}
$$

Moreover, this relation matches the relevant equation for isolated PAHs. ${ }^{47}$ Hence, our submatrix $\boldsymbol{G}_{(1)}^{(\alpha)}$ actually coincides with the respective matrix obtained previously. ${ }^{47}$ The same accordingly refers to their designations, where the superscript $(\alpha)$ indicates the dependence upon the perturbation $\alpha$. The meaning of the matrix $\boldsymbol{G}_{(1)}^{(\alpha)}$ will be discussed later in this section. Let us note only that this matrix represents the direct intersubset coupling within the DM $\tilde{\boldsymbol{P}}$.

As opposed to Eq. (18), the form of the second equation following from Eq. (12) depends on the position of electrophile. Moreover, it may be easily solved algebraically. ${ }^{64}$ The result takes the form

$$
\begin{aligned}
& \boldsymbol{g}_{(1)}^{*}=-\boldsymbol{F}_{e} \boldsymbol{v}_{(1)}^{*}=-\frac{1}{\sqrt{2}} \boldsymbol{F}_{e} \boldsymbol{a}, \\
& \mathbf{g}_{(1)}^{\mathrm{o}}=-\boldsymbol{F}_{e} \boldsymbol{v}_{(1)}^{\mathrm{o}}=-\frac{1}{\sqrt{2}} \boldsymbol{F}_{e} \boldsymbol{B} \boldsymbol{Q} \boldsymbol{a},
\end{aligned}
$$

where $\boldsymbol{g}_{(1)}^{*}$ and $\boldsymbol{g}_{(1)}^{\circ} \quad$ correspond to first order Hamiltonian matrices $\tilde{\boldsymbol{H}}_{(1)}^{*}$ and $\tilde{\boldsymbol{H}}_{(1)}^{\text {o }}$ and thereby to $\boldsymbol{H}_{(1)}^{*}$ and $\boldsymbol{H}_{(1)}^{\mathrm{o}}$ of Eq. (2), respectively, and $\boldsymbol{F}_{e}$ is an $n \times n$-dimensional square matrix, viz.

$$
\boldsymbol{F}_{e}=(\mathbf{I}+\mu \boldsymbol{R})^{-1} \boldsymbol{R}
$$

The subscript $e$ indicates the matrix $\boldsymbol{F}_{e}$ to represent the electrophile. As is seen from Eq. (19), the columnmatrices $\boldsymbol{g}_{(1)}^{*}$ and $\boldsymbol{g}_{(1)}^{\mathrm{o}}$ do not depend on the perturbation $\alpha$. It is no surprise in this connection that Eqs. (19) and (20) coincide with the relevant expressions ${ }^{64}$ referring to an $\mathrm{AH}$ interacting with an external electron-accepting subsystem.

Let the second order increment to the population of the orbital $\varphi_{(-) e}$ to be denoted by $q_{(-) e}^{(2)}$. As it follows immediately after substituting Eq. (17) into Eq. (11), the column-matrices $\boldsymbol{g}_{(1)}^{*}$ and $\boldsymbol{g}_{(1)}^{\text {o }}$ defined by Eq. (19) suffice to derive this increment. We then obtain 


$$
q_{(-) e}^{(2) *}=2 \boldsymbol{g}_{(1)}^{*+} \boldsymbol{g}_{(1)}^{*}, \quad q_{(-) e}^{(2) \circ}=2 \boldsymbol{g}_{(1)}^{\mathrm{o+}} \boldsymbol{g}_{(1)}^{\mathrm{o}}
$$

for the attacks upon AOs of subsets $\left\{\chi^{*}\right\}$ and $\left\{\chi^{\circ}\right\}$, respectively. Equation (21) along with Eqs. (19) and (20) indicates that the second order populations acquired by the electrophile are not influenced by the perturbation $\alpha$. Hence, these coincide with the relevant populations for the parent $\mathrm{AH}$ under attack of the same reagent. Use of Eq. (19) within Eq. (21) yields

$$
q_{(-) e}^{(2) *}=\boldsymbol{a}^{+} \mathbf{F}_{e}^{+} \mathbf{F}_{e} \boldsymbol{a}, \quad q_{(-) e}^{(2) o}=\boldsymbol{a}^{+} \mathbf{Q} \mathbf{B}^{+} \mathbf{F}_{e}^{+} \mathbf{F}_{e} \mathbf{B Q} \boldsymbol{a} .
$$

Let us recall now that the column- matrix a contains a single non-zero element equal to $v$ in its kth position if the kth $\mathrm{AO}\left(\chi_{k}^{*}\right.$ or $\left.\chi_{k}^{0}\right)$ is under attack of electrophile. Given that this is the case, diagonal elements $D_{(0) k k}^{*}$ and $D_{(0) k k}^{\mathrm{o}}$ of the following matrices

$$
\boldsymbol{D}_{(0)}^{*}=\boldsymbol{F}_{e}^{+} \boldsymbol{F}_{e}, \quad \boldsymbol{D}_{(0)}^{\circ}=\boldsymbol{Q B} \boldsymbol{B}^{+} \boldsymbol{F}_{e}^{+} \boldsymbol{F}_{e} \boldsymbol{B} \boldsymbol{Q}
$$

determine the relevant populations $q_{(-) e}^{(2) *}$ and $q_{(-) e}^{(2) o}$, where the subscript (0) indicates the zero order magnitude of these matrices with respect to the perturbation $\alpha$. Again, these elements depend on characteristics of both the reactant and the reagent (these are correspondingly contained within matrices $\boldsymbol{B}$ and $\boldsymbol{F}_{e}$ ). Hence, the elements $D_{(0) k k}^{*}$ and $D_{(0) k k}^{o}$ may be interpreted as charge transfer (CT) abilities of the system consisting of the parent $\mathrm{AH}$ and the electrophile for attacks of the latter upon AOs $\chi_{k}^{*}$ and $\chi_{k}^{\circ}$, respectively. Moreover, the same elements represent the zero order contributions to the respective $\mathrm{CT}$ abilities of the heteroatom-containing system. That is why $\boldsymbol{D}_{(0)}^{*}$ and $\boldsymbol{D}_{(0)}^{\mathrm{o}}$ will be referred to as zero order matrices of CT abilities. Furthermore, both $\boldsymbol{D}_{(0)}^{*}$ and $\boldsymbol{D}_{(0)}^{\circ}$ are positivedefinite matrices ${ }^{72}$ of the form $\boldsymbol{A}^{+} \boldsymbol{A}$. Thus, the electrophile always acquires an additional population within the zero order approximation in accordance with the expectation. From Eqs.(6) and (23) we obtain that

$$
\operatorname{SpurD}_{(0)}^{*}=\operatorname{SpurD}_{(0)}^{\circ},
$$

i.e. sums of zero order $\mathrm{CT}$ abilities over attacks upon AOs of subsets $\left\{\chi^{*}\right\}$ and $\left\{\chi^{\circ}\right\}$ coincide one with another.

To derive first order increments to the same CT abilities due to introduction of the heteroatom, let us turn to the third order correction to the population of electrophile $\left(q_{(-) e}^{(3)}\right)$. Third order analogues of Eqs. (21) and (22) may be derived from the last diagonal element of the submatrix $\tilde{\boldsymbol{P}}_{2(3)}$ of Eq. (11). It is seen that this submatrix contains the second order matrix $\boldsymbol{G}_{(2)}$ defined by Eq. (12) for $k=2$. Thus, let us turn to this matrix equation along with the definition of the matrix $\boldsymbol{V}_{(2)}$ of Eq. (15).

Substituting Eqs. (16) and (17) into Eq. (15) shows that the total matrix $\boldsymbol{V}_{(2)}$ also consists of a square $n \times n-$ dimensional matrix and of an attached columnmatrix. In analogy with the matrix $\boldsymbol{G}_{(1)}$ of Eq. (17), let these submatrices to be correspondingly denoted by $\boldsymbol{V}_{(2)}^{(\alpha)}$ and $\boldsymbol{v}_{(2)}$. The matrix $\boldsymbol{G}_{(2)}$ may be then accordingly sought in the form containing a square block $\boldsymbol{G}_{(2)}^{(\alpha)}$ and a column- matrix $\boldsymbol{g}_{(2)}$, i.e.

$$
\boldsymbol{G}_{(2)}=\left|\boldsymbol{G}_{(2)}^{(\alpha)} \quad \boldsymbol{g}_{(2)}\right| .
$$

Two consequences follow immediately after accepting Eq. (25): First, the expression for the third order correction to the population of the orbital $\varphi_{(-) e}$ contains the column-matrices $\boldsymbol{g}_{(1)}$ and $\boldsymbol{g}_{(2)}$ only, viz.

$$
q_{(-) e}^{(3)}=2\left(\boldsymbol{g}_{(1)}^{+} \boldsymbol{g}_{(2)}+\boldsymbol{g}_{(2)}^{+} \boldsymbol{g}_{(1)}\right) .
$$

Second, the relation of Eq. (12) for $k=2$ may be decomposed into two matrix equations determining the submatrices $\boldsymbol{G}_{(2)}^{(\alpha)}$ and $\boldsymbol{g}_{(2)}$ separately. It is evident that the relation conditioning the latter is sufficient for our purpose. As with the equation for $\boldsymbol{g}_{(1)}$, an algebraic solution may be obtained, viz.

$$
\boldsymbol{g}_{(2)}=-\boldsymbol{F}_{e} \boldsymbol{v}_{(2)},
$$

where the column-matrix $\boldsymbol{v}_{(2)}$ results from Eqs. (15), (16) and (17). We obtain

$$
\begin{aligned}
& \boldsymbol{v}_{(2)}^{*}=\frac{1}{2} \boldsymbol{A}_{(1)} \boldsymbol{g}_{(1)}^{*}-\frac{1}{\sqrt{2}} \boldsymbol{G}_{(1)}^{(\alpha)} \boldsymbol{Q} \boldsymbol{B}^{+} \boldsymbol{a}, \\
& \boldsymbol{v}_{(2)}^{\mathrm{o}}=\frac{1}{2} \boldsymbol{A}_{(1)} \boldsymbol{g}_{(1)}^{\mathrm{o}}+\frac{1}{\sqrt{2}} \boldsymbol{G}_{(1)}^{(\alpha)} \boldsymbol{a}
\end{aligned}
$$

for attacks upon AOs of subsets $\left\{\chi^{*}\right\}$ and $\left\{\chi^{\circ}\right\}$, respectively. It is seen that the right-hand sides of these expressions contain the perturbations $\alpha$ and $v$ in the form of products. The relevant corrections $q_{(-) e}^{(3) *}$ and $q_{(-) e}^{(3) o}$ following from Eq. (26) then accordingly depend on the product $\alpha v^{2}$ and thereby represent the result of the interplay between intra- ad intermolecular perturbations. Furthermore, two additive components are present within both $\boldsymbol{v}_{(2)}^{*}$ and $\boldsymbol{v}_{(2)}^{0}$. The first $\boldsymbol{A}_{(1)}$ containing contribution may be traced back to the increasing absolute value of the diagonal Hamiltonian matrix element referring to the NCMO $\Psi_{(+) 1}$ of the parent $\mathrm{AH}$ due to the intramolecular perturbation $\alpha$. Meanwhile, the second contributions are related to the intersubset coupling inside the $\mathrm{AH}$ represented by the matrix $\boldsymbol{G}_{(1)}^{(\alpha)}$. Owing to Eqs. (26) and (27), analogous 
two components reveal themselves within the columnmatrices $\boldsymbol{g}_{(2)}^{*}$ and $\boldsymbol{g}_{(2)}^{\mathbf{o}}$, as well as within third order populations $q_{(-) e}^{(3) *}$ and $q_{(-) e}^{(3) \circ}$.

The matrix $\boldsymbol{G}_{(1)}^{(\alpha)}$ was shown to give birth to the socalled intersubset polarization matrices of PAHs $\left(\boldsymbol{X}_{(1)}^{(\alpha)}\right.$ and $\left.\boldsymbol{Z}_{(1)}^{(\alpha)}\right)$ in accordance with the following relations ${ }^{47}$

$$
\begin{aligned}
& \boldsymbol{X}_{(1)}^{(\alpha)}=-2 \boldsymbol{B} \boldsymbol{Q} \boldsymbol{G}_{(1)}^{(\alpha)+}=-2 \boldsymbol{G}_{(1)}^{(\alpha)} \boldsymbol{Q} \boldsymbol{B}^{+}, \\
& \boldsymbol{Z}_{(1)}^{(\alpha)}=2 \boldsymbol{G}_{(1)}^{(\alpha)+} \boldsymbol{B} \boldsymbol{Q}=2 \boldsymbol{Q} \boldsymbol{B}^{+} \boldsymbol{G}_{(1)}^{(\alpha)} .
\end{aligned}
$$

Thus, recalling of the meaning and of principal properties of matrices $\boldsymbol{X}_{(1)}^{(\alpha)}$ and $\boldsymbol{Z}_{(1)}^{(\alpha)}$ becomes appropriate when looking for a more illustrative interpretation of the $\boldsymbol{G}_{(1)}^{(\alpha)}$-containing components of the aboveindicated characteristics.

Let us note first that matrices $\boldsymbol{X}_{(1)}^{(\alpha)}$ and $\boldsymbol{Z}_{(1)}^{(\alpha)}$ take diagonal positions referring to subsets of AOs $\left\{\chi^{*}\right\}$ and $\left\{\chi^{\circ}\right\}$, respectively, within the first order contribution of the charge- bond order (CBO) matrix of PAHs. ${ }^{47}$ Consequently, diagonal elements of these matrices determine the actual populations inside the hydrocarbon fragment of an $\mathrm{AH}$ after introducing a heteroatom (note that all populations coincide with unity within an $\left.\mathrm{AH}^{49,50}\right)$. Furthermore, the matrices $\boldsymbol{X}_{(1)}^{(\alpha)}$ and $\boldsymbol{Z}_{(1)}^{(\alpha)}$ were shown to be connected by an unitary transformation, viz.

$$
\boldsymbol{X}_{(1)}^{(\alpha)}=-\boldsymbol{B} \boldsymbol{Q} \boldsymbol{Z}_{(1)}^{(\alpha)} \boldsymbol{Q B} \boldsymbol{B}^{+}
$$

Using Eq. (6), we then obtain

$$
\operatorname{Spur}_{(1)}^{(\alpha)}=-\operatorname{Spur}_{(1)}^{(\alpha)} .
$$

Thus, total excessive populations over subsets $\left\{\chi^{*}\right\}$ and $\left\{\chi^{\circ}\right\}$ of PAHs are of opposite signs and of coinciding absolute values so that their sum vanishes. Finally, matrices $\boldsymbol{X}_{(1)}^{(\alpha)}$ and $\boldsymbol{Z}_{(1)}^{(\alpha)}$ proved to be representable ${ }^{47}$ in the forms $\boldsymbol{A A}^{+}$and $-\boldsymbol{A} \boldsymbol{A}^{+}$and thereby were concluded to be positive-definite and negative-definite matrices ${ }^{72}$, respectively. Consequently, populations of all AOs $\chi_{k}^{*}$ were expected to grow, whilst those of all AOs $\chi_{k}^{\circ}$ were predicted to be reduced as a result of the perturbation $\alpha$. This conclusion is nothing more than the rule of the alternating polarity.

Before returning to Eqs. (26)-(28) it deserves emphasizing that both the results shown in Eqs. (29)-(31) and the discussion nearby are directly transferable to the case of PAHs under attack of electrophile. The reason for that consists in the independent action of perturbations $\alpha$ and $v$ within first order Hamiltonian matrices as discussed just after Eq. (9). For illustration, we exhibit below the first order contribution $\boldsymbol{P}_{(1)}^{*}$ to the total CBO matrix of PAHs under attack of electrophile upon an $\mathrm{AO}$ of the subset $\left\{\chi^{*}\right\}$ following after retransformation ${ }^{47}$ of the DM $\tilde{\boldsymbol{P}}^{*}$ into the basis of AOs. This correction takes the form

$$
\boldsymbol{P}_{(1)}^{*}=\left|\begin{array}{ccc}
\boldsymbol{X}_{(1)}^{(\alpha)} & \mathbf{0} & -\sqrt{2} \boldsymbol{g}_{(1)}^{*} \\
\mathbf{0} & \boldsymbol{Z}_{(1)}^{(\alpha)} & -\sqrt{2} \boldsymbol{Q} \boldsymbol{B}^{+} \boldsymbol{g}_{(1)}^{*} \\
-\sqrt{2} \boldsymbol{g}_{(1)}^{*+} & -\sqrt{2} \boldsymbol{g}_{(1)}^{*+} \boldsymbol{B} \boldsymbol{Q} & 0
\end{array}\right|
$$

and contains the intersubset polarization matrices $\boldsymbol{X}_{(1)}^{(\alpha)}$ and $\boldsymbol{Z}_{(1)}^{(\alpha)}$ in its diagonal positions.

It is evident that relations of Eq. (29) allow the $\boldsymbol{G}_{(1)}^{(\alpha)}$-containing terms of Eqs. (26)-(28) to be reformulated into those depending on the intersubset polarization matrices $\boldsymbol{X}_{(1)}^{(\alpha)}$ and $\boldsymbol{Z}_{(1)}^{(\alpha)}$. This implies the relevant components of corrections $q_{(-) e}^{(3) *}$ and $q_{(-) e}^{(3) o}$ to be related to excessive populations of the isolated reactant. To perform the relevant procedure, Eq. (6) also should be invoked. Thereupon, Eqs. (19), (20), (27) and (28) should be substituted into Eq. (26). We then obtain

$$
\begin{aligned}
& q_{(-) e}^{(3) *}=a^{+}\left(M_{(1)}^{*}+N_{(1)}^{*}\right) a, \\
& q_{(-) e}^{(3) o}=a^{+}\left(M_{(1)}^{o}+N_{(1)}^{o}\right) a,
\end{aligned}
$$

where $n \times n$-dimensional square matrices of the righthand sides take the form

$$
\begin{aligned}
\boldsymbol{M}_{(1)}^{*}= & \frac{1}{2}\left(\boldsymbol{F}_{e}^{+} \boldsymbol{F}_{e} \boldsymbol{X}_{(1)}^{(\alpha)}+\boldsymbol{X}_{(1)}^{(\alpha)} \boldsymbol{F}_{e}^{+} \boldsymbol{F}_{e}\right), \\
\boldsymbol{N}_{(1)}^{*}= & -\frac{1}{2}\left(\boldsymbol{F}_{e}^{+} \boldsymbol{F}_{e} \boldsymbol{A}_{(1)} \boldsymbol{F}_{e}+\boldsymbol{F}_{e}^{+} \boldsymbol{A}_{(1)} \boldsymbol{F}_{e}^{+} \boldsymbol{F}_{e}\right), \\
\boldsymbol{M}_{(1)}^{\mathrm{o}}= & \frac{1}{2}\left(\boldsymbol{Q B} \boldsymbol{B}^{+} \boldsymbol{F}_{e}^{+} \boldsymbol{F}_{e} \boldsymbol{B} \boldsymbol{Q} \boldsymbol{Z}_{(1)}^{(\alpha)}+\right. \\
& \left.\boldsymbol{Z}_{(1)}^{(\alpha)} \boldsymbol{Q} \boldsymbol{B}^{+} \boldsymbol{F}_{e}^{+} \boldsymbol{F}_{e} \boldsymbol{B} \boldsymbol{Q}\right), \\
\boldsymbol{N}_{(1)}^{\mathrm{o}}= & -\frac{1}{2} \boldsymbol{Q} \boldsymbol{B}^{+}\left(\boldsymbol{F}_{e}^{+} \boldsymbol{F}_{e} \boldsymbol{A}_{(1)} \boldsymbol{F}_{e}+\right. \\
& \left.\boldsymbol{F}_{e}^{+} \boldsymbol{A}_{(1)} \boldsymbol{F}_{e}^{+} \boldsymbol{F}_{e}\right) \boldsymbol{B} \boldsymbol{Q} .
\end{aligned}
$$

The contributions $\boldsymbol{M}_{(1)}^{*}$ and $\boldsymbol{M}_{(1)}^{o}$ originate from the intersubset coupling in the parent $\mathrm{AH}$ due to the perturbation $\alpha$ and contain the intersubset polarization matrices $\boldsymbol{X}_{(1)}^{(\alpha)}$ and $\boldsymbol{Z}_{(1)}^{(\alpha)}$ in accordance with the above discussion. Meanwhile, the remaining increments (viz. $\boldsymbol{N}_{(1)}^{*}$ and $\boldsymbol{N}_{(1)}^{\mathrm{o}}$ ) depend on the submatrix $\frac{1}{2} \boldsymbol{A}_{(1)}$ and are related to the first component of the column-matrices $\boldsymbol{v}_{(2)}^{*}$ and $\boldsymbol{v}_{(2)}^{\circ}$ of Eq. (28). The subscript (1) of the lefthand sides of Eqs. (34)-(37) indicates the matrices concerned to be first order terms with respect to the perturbation $\alpha$. 
Finally, comparison of Eq. (33) to Eq. (22) indicates these expressions to be of similar constitution. This allows us to define new matrices $\boldsymbol{D}_{(1)}^{*}$ and $\boldsymbol{D}_{(1)}^{\circ}$, diagonal elements of which represent first order increments to the above-discussed CT abilities, viz.

$$
\boldsymbol{D}_{(1)}^{*}=\boldsymbol{M}_{(1)}^{*}+\boldsymbol{N}_{(1)}^{*}, \quad \boldsymbol{D}_{(1)}^{\mathrm{o}}=\boldsymbol{M}_{(1)}^{\mathrm{o}}+\boldsymbol{N}_{(1)}^{\mathrm{o}} .
$$

The principal properties of these matrices are studied in the next Section.

\section{ANALYSIS OF EXPRESSIONS OBTAINED}

Our analysis will consist of three steps. Let us dwell first on results that may be obtained without additional approximations, except for the above-described perturbation theory. Let us start with components $\boldsymbol{M}_{(1)}^{*}$ and $\boldsymbol{M}_{(1)}^{\mathrm{o}}$. From Eq. (34) it follows that

$$
\operatorname{Spur}_{(1)}^{*}=\operatorname{Spur}\left(\boldsymbol{F}_{e} \boldsymbol{X}_{(1)}^{(\alpha)} \boldsymbol{F}_{e}^{+}\right)>0
$$

where the positive-definite nature ${ }^{47}$ of the matrix $\boldsymbol{X}_{(1)}^{(\alpha)}$ is taken into consideration. It is seen that the sum of CT abilities of our system over attacks upon AOs of the subset $\left\{\chi^{*}\right\}$ becomes increased owing to the increment $\boldsymbol{M}_{(1)}^{*}$. Using Eqs.(6), (30) and (36), we accordingly obtain

$$
\operatorname{Spur}_{(1)}^{o}=-\operatorname{Spur}\left(\boldsymbol{F}_{e} \boldsymbol{X}_{(1)}^{(\alpha)} \boldsymbol{F}_{e}^{+}\right)<0 .
$$

Thus, the matrix $\boldsymbol{M}_{(1)}^{\mathrm{o}}$ contributes to lowering of the sum of CT abilities over attacks upon AOs of the subset $\left\{\chi^{\circ}\right\}$. Finally, Eqs. (39) and (40) yield

$$
\operatorname{SpurM}_{(1)}^{*}=-\operatorname{Spur} \boldsymbol{M}_{(1)}^{\mathrm{o}} .
$$

Hence, the intersubset coupling in an $\mathrm{AH}$ gives rise to alterations of coinciding absolute values and of opposite signs in the above-specified sums of CT abilities as it was the case with sums of excessive populations in the isolated reactant (see Eq. (31)). Moreover, the following relation

$$
\boldsymbol{M}_{(1)}^{*}=-\boldsymbol{B} \boldsymbol{Q} \boldsymbol{M}_{(1)}^{\mathrm{o}} \boldsymbol{Q} \boldsymbol{B}^{+}
$$

results from Eqs. (30), (34) and (36) and it resembles that of Eq. (30). In this connection, a certain parallelism may be expected between alterations in the CT abilities for attacks upon separate AOs due to the intersubset coupling and the excessive populations of the same AOs in the isolated reactant. This parallelism is likely to embrace alternation of corrections over the hydrocarbon chain. These anticipations are verified below in this section.

Let us turn now to matrices $\boldsymbol{N}_{(1)}^{*}$ and $\boldsymbol{N}_{(1)}^{\mathrm{o}}$ defined by Eqs. (35) and (37). After employment of Eq. (6), we obtain

$$
\begin{aligned}
\operatorname{SpurN}_{(1)}^{*} & =\operatorname{SpurN}_{(1)}^{\mathrm{o}}= \\
& =-\operatorname{Spur}\left[\left(\boldsymbol{F}_{e}^{+}\right)^{3 / 2} \boldsymbol{A}_{(1)}\left(\boldsymbol{F}_{e}\right)^{3 / 2}\right]<0
\end{aligned}
$$

and

$$
\boldsymbol{N}_{(1)}^{*}=\boldsymbol{B} \boldsymbol{Q} \boldsymbol{N}_{(1)}^{\mathrm{o}} \boldsymbol{Q B} \boldsymbol{B}^{+} .
$$

The Hermitian (symmetric) and positive-definite nature of the matrix $\boldsymbol{F}_{e}$ of Eq. (20) is additionally used when deriving Eq. (43). (Note that unique positive-definite square roots may always be defined for Hermitian positive-definite matrices ${ }^{72}$ ). The Hermitian property for the matrix $\boldsymbol{F}_{e}$, in turn, easily follows from the equality $\boldsymbol{R}=\boldsymbol{R}^{+}$along with the standard definition of a power function of any Hermitian matrix in terms of its spectrum $^{73,74}$. The reasoning behind the positivedefinite nature of the matrix $\boldsymbol{F}_{e}$ is as follows: The matrix product $\boldsymbol{B B}^{+}$was concluded to be a positivedefinite matrix for the most common case of even AHs of a finite size ${ }^{48}$. The same accordingly refers to the positive square root $\left(\boldsymbol{B} \boldsymbol{B}^{+}\right)^{1 / 2}$, the latter coinciding with the inverse counterpart $\left(\boldsymbol{R}^{-1}\right)$ of the matrix $\boldsymbol{R}$ as Eq. (8) indicates. Further, $\boldsymbol{F}_{e}^{-1}$ actually takes the form of the sum of the positive increment $\mu \boldsymbol{I}$ and of the matrix $\boldsymbol{R}^{-1}$ (see Eq. (20)). Hence, $\boldsymbol{F}_{e}^{-1}$ also is a positive-definite matrix for the above-specified AHs. Finally, the positive-definite nature of a certain matrix is known to be preserved when passing to its inverse counterpart ${ }^{72}$. Thus, the very matrix $\boldsymbol{F}_{e}$ also may be concluded to be a positive-definite one.

Let us return to Eqs. (43) and (44). It is seen that alterations in the sums of CT abilities over attacks upon AOs of subsets $\left\{\chi^{*}\right\}$ and $\left\{\chi^{\circ}\right\}$ coincide one with another in this case. Moreover, these alterations prove to be negative quantities. Hence, the CT abilities for attacks upon individual AOs also may be anticipated to be predominantly reduced owing to the increments $\boldsymbol{N}_{(1)}^{*}$ amd $\boldsymbol{N}_{(1)}^{\mathrm{o}}$. This expectation evidently is in line with the origin of matrices $\boldsymbol{N}_{(1)}^{*}$ and $\boldsymbol{N}_{(1)}^{\circ}$, namely with the growing absolute value of the diagonal Hamiltonian matrix element referring to the NCMO $\Psi_{(+) 1}$ due to the perturbation $\alpha$. Indeed, such a growth implies an increase of the distance between one of the electrondonating orbitals of the reactant (namely $\Psi_{(+) 1}$ ) and the electron-accepting orbital $\left(\varphi_{(-) e}\right)$ in the energy scale.

The second step of our analysis consists in evaluation of separate elements of matrices $\boldsymbol{M}_{(1)}^{*}, \boldsymbol{M}_{(1)}^{\mathrm{o}}, \boldsymbol{N}_{(1)}^{*}$ and $\boldsymbol{N}_{(1)}^{\mathrm{o}}$. To this end, we will invoke the so-called diagonally-dominant nature of positive- 
definite matrices along with positive signs of the diagonal elements themselves ${ }^{72}$ (This property allows us to consider the matrix concerned, e.g. $\boldsymbol{F}_{e}^{+} \boldsymbol{F}_{e}$, as a nearly diagonal matrix). Let us start with matrices $\boldsymbol{M}_{(1)}^{*}$ and $\boldsymbol{M}_{(1)}^{\mathrm{o}}$. Elements $X_{(1) k l}^{(\alpha)}$ of the matrix $\boldsymbol{X}_{(1)}^{(\alpha)}$ were shown to be of similar absolute values except for the single element $X_{(1) 11}^{(\alpha)}$ referring to the site ${ }^{47}$ of the perturbation $\alpha$. If the latter case is excluded from consideration, from Eq. (34) it follows that

$$
M_{(1) k k}^{*} \cong\left(\boldsymbol{F}_{e}^{+} \boldsymbol{F}_{e}\right)_{k k} X_{(1) k k}^{(\alpha)}>0 .
$$

Hence, corrections to $\mathrm{CT}$ abilities for attacks upon AOs $\chi_{k}^{*}(k \neq 1)$ due to the intersubset coupling are positive quantities in the framework of our approximation. Moreover, these corrections are proportional to respective (positive ${ }^{47}$ ) excessive populations in the isolated reactant $\left(X_{(1) k k}^{(\alpha)}>0\right)$ and to the relevant CT abilities of the parent-hydrocarbon-containing system $D_{(0) k k}^{*}$ (see Eq. (23) for the definition of the latter). From Eq. (36), we accordingly obtain

$$
M_{(1) k k}^{\mathrm{o}} \cong\left(\boldsymbol{Q B} \boldsymbol{B}^{+} \boldsymbol{F}_{e}^{+} \boldsymbol{F}_{e} \boldsymbol{B} \boldsymbol{Q}\right)_{k k} Z_{(1) k k}^{(\alpha)}<0,
$$

where similar absolute values ${ }^{47}$ of elements $Z_{(1) k l}^{(\alpha)}$ also are taken into consideration. Proportionality of the correction $M_{(1) k k}^{\mathrm{o}}$ to the relevant (negative) excessive population of the isolated reactant $\left(Z_{(1) k k}^{(\alpha)}<0\right)$ is seen from Eq. (46) along with that between $M_{(1) k k}^{\circ}$ and the zero order CT ability for the attack upon the $\mathrm{AO} \chi_{k}^{\circ}$ in the parent $\mathrm{AH}$. Thus, the above-anticipated alternation of corrections to the $\mathrm{CT}$ abilities originating from the intersubset coupling in the reactant is now additionally supported.

A similar analysis may be carried out also for the remaining matrices $\boldsymbol{N}_{(1)}^{*}$ and $\boldsymbol{N}_{(1)}^{\mathrm{o}}$. The relevant diagonal elements may be approximated as follows

$$
\begin{aligned}
N_{(1) k k}^{*} & =-\alpha\left(\boldsymbol{F}_{e}^{+} \boldsymbol{F}_{e}\right)_{k 1}\left(\boldsymbol{F}_{e}\right)_{1 k} \cong \\
& \cong-\alpha\left[\left(\boldsymbol{F}_{e}\right)_{k k}+\left(\boldsymbol{F}_{e}\right)_{11}\right]\left[\left(\boldsymbol{F}_{e}\right)_{1 k}\right]^{2}<0, \\
N_{(1) k k}^{\mathrm{o}} & \cong-\alpha\left[(\boldsymbol{B} \boldsymbol{Q})_{1 k}\right]^{2}\left(\boldsymbol{F}_{e}^{+} \boldsymbol{F}_{e}\right)_{11}\left(\boldsymbol{F}_{e}\right)_{11}<0
\end{aligned}
$$

and are negative quantities for both subsets. Thus, any CT ability of our system is predicted to be suppressed owing to increments concerned in accordance with our previous expectation.

The final effect of heteroatom of the reactant upon individual CT abilities of the whole system follows after summing up the two principal components as Eq. (38) indicates. For the attack upon an AO $\chi_{k}^{\circ}$ of the subset $\left\{\chi^{\circ}\right\}$, two negative increments seem to be added together, and a suppression of the relevant total CT ability is consequently expected. Meanwhile, the effect of the perturbation $\alpha$ upon the CT ability referring to an $\mathrm{AO} \chi_{k}^{*}$ of the heteroatom-containing subset $\left\{\chi^{*}\right\}$ depends on relative absolute values of the two increments of opposite signs. This implies the subset $\left\{\chi^{*}\right\}$ to be of a particular interest.

In this connection, let us turn to the principal (third) step of our analysis devoted to the case of the socalled soft electrophiles ${ }^{10,29}$. In accordance with the high electron-accepting ability of these electrophiles, let us assume that the parameter $\mu$ to take a sufficiently small value so that the matrix $\boldsymbol{F}_{e}$ of Eq. (20) may be approximated by $\boldsymbol{R}$. [The nature of this approximation and conditions of its validity are discussed later in this section]. Given that this is the case, the suppressive increments to CT abilities following from matrices $\boldsymbol{N}_{(1)}^{*}$ and $\boldsymbol{N}_{(1)}^{\mathrm{o}}$ also may be related to the intersubset polarization matrices $\boldsymbol{X}_{(1)}^{(\alpha)}$ and $\boldsymbol{Z}_{(1)}^{(\alpha)}$. Consequently, a direct comparison of the principal contributions to $\mathrm{CT}$ abilities becomes possible.

To demonstrate this, let us start with Eq. (35). After invoking the above-mentioned approximation, we obtain

$$
\overline{\boldsymbol{N}}_{(1)}^{*}=-\frac{1}{2} \boldsymbol{R}\left(\boldsymbol{R} \boldsymbol{A}_{(1)}+\boldsymbol{A}_{(1)} \boldsymbol{R}\right) \boldsymbol{R},
$$

where overlined characteristics here and below refer to the case of small $\mu$ values. Let us take now the relation of Eq. (18) and multiply it by $\mathbf{B}^{+}$from its right-hand side. A subsequent use of Eqs. (6) and (29) then yields

$$
\boldsymbol{R} \boldsymbol{A}_{(1)}=\boldsymbol{X}_{(1)}^{(\alpha)}-2 \boldsymbol{R} \boldsymbol{G}_{(1)}^{(\alpha)} \boldsymbol{B}^{+} .
$$

Let us substitute the above relation and its Hermitianconjugate counterpart into Eq. (49) and replace the product $\boldsymbol{R} \boldsymbol{B}$ by $\boldsymbol{B} \boldsymbol{Q}$ in accordance with Eq. (5). After an additional employment of Eq. (29), we obtain

$$
\overline{\boldsymbol{N}}_{(1)}^{*}=-\frac{1}{2}\left(\boldsymbol{R}^{2} \boldsymbol{X}_{(1)}^{(\alpha)}+\boldsymbol{X}_{(1)}^{(\alpha)} \boldsymbol{R}^{2}+2 \boldsymbol{R} \boldsymbol{X}_{(1)}^{(\alpha)} \boldsymbol{R}\right)
$$

Thus, the matrix $\overline{\boldsymbol{N}}_{(1)}^{*}$ becomes expressed via the matrix $\boldsymbol{X}_{(1)}^{(\alpha)}$. An analogous procedure for the matrix $\overline{\boldsymbol{N}}_{(1)}^{\mathrm{o}}$ yields an expression in terms of $\boldsymbol{Z}_{(1)}^{(\alpha)}$, viz.

$$
\overline{\boldsymbol{N}}_{(1)}^{\mathrm{o}}=\frac{1}{2}\left(\boldsymbol{Q}^{2} \boldsymbol{Z}_{(1)}^{(\alpha)}+\boldsymbol{Z}_{(1)}^{(\alpha)} \boldsymbol{Q}^{2}+2 \boldsymbol{Q} \boldsymbol{Z}_{(1)}^{(\alpha)} \boldsymbol{Q}\right) .
$$

Meanwhile, replacement of the matrix $\boldsymbol{F}_{e}$ by $\boldsymbol{R}$ within Eqs. (34) and (36) yields

$$
\overline{\boldsymbol{M}}_{(1)}^{*}=\frac{1}{2}\left(\boldsymbol{R}^{2} \boldsymbol{X}_{(1)}^{(\alpha)}+\boldsymbol{X}_{(1)}^{(\alpha)} \boldsymbol{R}^{2}\right)
$$




$$
\overline{\boldsymbol{M}}_{(1)}^{\mathrm{o}}=\frac{1}{2}\left(\boldsymbol{Q}^{2} \boldsymbol{Z}_{(1)}^{(\alpha)}+\boldsymbol{Z}_{(1)}^{(\alpha)} \boldsymbol{Q}^{2}\right),
$$

where Eq. (6) is additionally used when deriving Eq. (54).

Comparison of formulae shown in Eqs. (51) and (53) and referring to the most intriguing subset $\left\{\chi^{*}\right\}$ indicates that the first two contributions of their righthand sides cancel out each other when building up the total matrix $\overline{\boldsymbol{D}}_{(1)}^{*}$ in accordance with Eq. (38). As a result, this matrix is determined by the last increment of Eq. (51), viz.

$$
\overline{\boldsymbol{D}}_{(1)}^{*}=-\boldsymbol{R} \boldsymbol{X}_{(1)}^{(\alpha)} \boldsymbol{R} .
$$

Representability ${ }^{47}$ of the matrix $\boldsymbol{X}_{(1)}^{(\alpha)}$ in the for $\boldsymbol{A A}^{+}$ along with the equality $\boldsymbol{R}=\boldsymbol{R}^{+}$then ensures the negativedefinite nature of the matrix $\overline{\boldsymbol{D}}_{(1)}^{*}$. Hence, the diagonal elements $\bar{D}_{(1) k k}^{*}$ are negative quantities for any $k$. Consequently, all CT abilities of our system referring to attacks of a soft electrophile upon AOs of the subset $\left\{\chi^{*}\right\}$ prove to be reduced after introduction of the heteroatom in spite of increased excessive populations. A side conclusion here is that the suppressive increment predominates over the alternating one in the total first order corrections to $\mathrm{CT}$ abilities for attacks upon AOs $\chi_{k}^{*}$ in the case of a soft electrophile.

For the subset $\left\{\chi^{\circ}\right\}$, all terms of Eqs. (52) and (54) are added together and we obtain

$$
\overline{\boldsymbol{D}}_{(1)}^{\mathrm{o}}=\boldsymbol{Q}^{2} \boldsymbol{Z}_{(1)}^{(\alpha)}+\boldsymbol{Z}_{(1)}^{(\alpha)} \boldsymbol{Q}^{2}+\boldsymbol{Q} \boldsymbol{Z}_{(1)}^{(\alpha)} \boldsymbol{Q}
$$

Matrices $\boldsymbol{R}$ and $\boldsymbol{Q}$ of AHs were shown to be expressible in the form of power series ${ }^{48}$. Moreover, zero order members of these series $\left(\boldsymbol{R}^{(0)}\right.$ and $\left.\boldsymbol{Q}^{(0)}\right)$ are proportional to the unit matrix $(\boldsymbol{I})$ of the respective dimension, i.e.

$$
\boldsymbol{R}^{(0)}=m \boldsymbol{I}, \quad \boldsymbol{Q}^{(0)}=n \boldsymbol{I},
$$

where $m$ and $n$ are numerical parameters and $m \cong n$. Let us substitute these approximate expressions into Eqs. (55) and (56). From Eq. (55) we then obtain

$$
\bar{D}_{(1) k k}^{*(0)}=-m^{2} X_{(1) k k}^{(\alpha)}<0 .
$$

This relation indicates that the higher is the excessive population of the AO $\chi_{k}^{*}$ in the isolated reactant, the more suppressed is the relevant CT ability of the whole system in the case of a soft electrophile. Meanwhile, from Eq. (56) it follows that

$$
\bar{D}_{(1) k k}^{(0)}=3 n^{2} Z_{(1) k k}^{(\alpha)}<0 .
$$

Thus, the CT ability is predicted to be lowered even more substantially if the AO $\chi_{k}^{0}$ is under attack. This result is in accordance with our previous expectations.

Before finishing this section, let us dwell on our principal approximation $\boldsymbol{F}_{e} \cong \boldsymbol{R}$ and conditions of its validity. It is evident that this approximation is equivalent to confinement to the zero order member $(\boldsymbol{I})$ of the power series expansion for the matrix $(\boldsymbol{I}+\mu \boldsymbol{R})^{-1}$, i.e.

$$
(\boldsymbol{I}+\mu \boldsymbol{R})^{-1}=\boldsymbol{I}-\mu \boldsymbol{R}+\mu^{2} \boldsymbol{R}^{2}+\ldots
$$

Thus, convergence of the above series is a necessary condition for its validity. It is also obvious that choice of a sufficiently small $\mu$ value (as discussed above) playes the decisive role in attempts to ensure such a convergence. Again, properties of the specific matrix $\boldsymbol{R}$ also seem to be important. To examine the last point in a more detail, let us turn to the standard definition of a power function of any Hermitian matrix in terms of its spectrum $^{73,74}$ and apply it to matrices $\boldsymbol{R}$ and $(\boldsymbol{I}+\mu \boldsymbol{R})^{-1}$. We may then conclude these two matrices to possess a common set of eigenvectors. Moreover, the relevant eigenvalues are interrelated, viz. an eigenvalue $\left(1+\mu r_{i}\right)^{-1}$ of the latter matrix corresponds to any eigenvalue $r_{i}$ of the parent matrix $\boldsymbol{R}$. Convergence of the series of Eq. (60) is then ensured if the expansions

$$
\left(1+\mu r_{i}\right)^{-1}=1-\left(\mu r_{i}\right)+\left(\mu r_{i}\right)^{2}+\ldots
$$

converge for any $i$. The relevant condition is known to coincide with the inequality $\left|\mu r_{i}\right|<1$. Let us recall now that both $\mu$ and $r$ are positive quantities (positive signs of eigenvalues $r$ result from the positive-definite nature of the matrix ${ }^{i} \boldsymbol{R}$ discussed above). Thus, the higher is the eigenvalue $r$, the narrower is the interval of $\mu$ values, wherein the requirement $\left|\mu r_{i}\right|<1$ is met and thereby convergence of Eq. (61) is ensured. This implies the highest eigenvalue of the given matrix $\boldsymbol{R}$ to play the decisive role in determining the actual interval of $\mu$ values, wherein the matrix expansion of Eq. (60) converges. It is evident that a sufficiently rapid convergence is additionally required to ensure a good quality of our approximation $\boldsymbol{F}_{e} \cong \boldsymbol{R}$. Nevertheless, the above-specified eigenvalue may be considered as a certain criterion of such a quality for a definite $\mu$ value inside the convergence interval of the matrix series of Eq. (60).

Let us note now that the matrix $\boldsymbol{R}^{-1}$ possesses eigenvalues $r_{i}^{-1}$ for the above-described reasons. Again, eigenvalues of the inverse matrix $\boldsymbol{R}^{-1}$ coincide with energies of occupied MOs of the parent hydrocarbon in our negative energy units. (The ultimate diagonalization of the block-diagonal Hamiltonian matrix of Eq. (7) yields MOs and their energies of the given $\mathrm{AH}$ ). Thus, 
absence of occupied (bonding) MOs described by oneelectron energies of low absolute values in the oneelectron spectrum of the given hydrocarbon (e.g. of those close to zero) may be considered as the first sign of validity of our approximation within a sufficiently wide interval of $\mu$ values. Since the above-specified absolute values generally become lower for more extended hydrocarbons, the interval of $\mu$ values concerned is likely to be accordingly reduced.

\section{ILLUSTRATION OF THE RESULTS}

As already mentioned, the pyridine molecule (Scheme 1) is a typical example when populations of $2 p_{z}$ AOs of the isolated reactant fail to predict relative reactivities of carbon atoms towards electrophiles. Indeed, increased populations of AOs of meta-positioned atoms $\left(\chi_{2}^{*}\right.$ and $\left.\chi_{3}^{*}\right) v s$. those of benzene follow from the rule of the alternating polarity ${ }^{45-47}$ in this case in contrast to suppressed reactivities determined experimentally.,8 Moreover, invoking of the HOMO/LUMO concept ${ }^{22-27}$ also is of a limited success in this case: Constitution of the HOMO of pyridine contains zero increments of AOs of both nitrogen atom and para-positioned carbon atom along with similar contributions of ortho- and metapositioned atoms ${ }^{75}$ and this pattern is not in line with the fact that exclusively meta-derivatives result from the relevant electrophilic substitution. In this connection, we will illustrate our general results by applying them just to pyridine under attack of electrophile in this section.

Numbering of $2 p_{z}$ AOs of this heterocycle (see Scheme 1) is chosen so as to ensure the anti-blockdiagonal structure of the relevant submatrix of the total Hamiltonian matrix of Eq. (1). [Non-zero resonance parameters referring to chemical bonds and equal to unit are found in the off-diagonal blocks $\boldsymbol{B}$ and $\boldsymbol{B}^{+}$in this matrix]. Matrices $\boldsymbol{B}, \boldsymbol{Q B}^{+}$and $\boldsymbol{X}_{(1)}^{(\alpha)}$ representing the pyridine heterocycle take the form ${ }^{47}$

$$
\begin{aligned}
& \boldsymbol{B}=\left|\begin{array}{lll}
1 & 0 & 1 \\
1 & 1 & 0 \\
0 & 1 & 1
\end{array}\right|, \quad \boldsymbol{Q B ^ { + }}=\frac{1}{3}\left|\begin{array}{ccc}
2 & 2 & -1 \\
-1 & 2 & 2 \\
2 & -1 & 2
\end{array}\right|, \\
& \boldsymbol{X}_{(1)}^{(\alpha)}=\frac{\alpha}{108}\left|\begin{array}{ccc}
43 & -5 & -5 \\
-5 & 1 & 1 \\
-5 & 1 & 1
\end{array}\right| .
\end{aligned}
$$

Meanwhile, matrices $\boldsymbol{R}$ and $\boldsymbol{R}^{-1}$ are as follows

$$
\boldsymbol{R}=\frac{1}{6}\left|\begin{array}{ccc}
5 & -1 & -1 \\
-1 & 5 & -1 \\
-1 & -1 & 5
\end{array}\right|, \quad \boldsymbol{R}^{-1}=\frac{1}{3}\left|\begin{array}{lll}
4 & 1 & 1 \\
1 & 4 & 1 \\
1 & 1 & 4
\end{array}\right|
$$

and illustrate validity of approximations of Eq. (57).

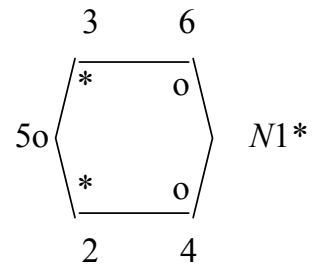

Scheme 1. Numbering of $2 \mathrm{p}_{z}$ AOs of pyridine. Atoms containing AOs of subsets $\left\{\chi^{*}\right\}$ and $\left\{\chi^{\circ}\right\}$ are accordingly denoted by $*$ and ${ }^{\mathrm{O}}$

[Note that $\boldsymbol{Q}=\boldsymbol{R}$ for this particular system].

Let us start our discussion with a notation that graphs representing matrices $\boldsymbol{R}$ and $\boldsymbol{R}^{-1}$ of Eq. (63) coincide with symmetric triangles. Consequently, these matrices may be diagonalized by the following transformation matrix

$$
\boldsymbol{U}=\left|\begin{array}{ccc}
\frac{1}{\sqrt{3}} & -\frac{2}{\sqrt{6}} & 0 \\
\frac{1}{\sqrt{3}} & \frac{1}{\sqrt{6}} & \frac{1}{\sqrt{2}} \\
\frac{1}{\sqrt{3}} & \frac{1}{\sqrt{6}} & -\frac{1}{\sqrt{2}}
\end{array}\right|
$$

that contains the relevant eigenvectors in its columns. As a result, eigenvalues of the matrix $\boldsymbol{R}$ may be easily shown to coincide with $\{1 / 2,1,1\}$, whilst those of $\boldsymbol{R}^{-1}$ are accordingly equal to $\{2,1,1\}$. Positive signs of these eigenvalues illustrate the positive-definite nature of matrices $\boldsymbol{R}$ and $\boldsymbol{R}^{-1}$ discussed above. Moreover, the largest eigenvalue of the matrix $\boldsymbol{R}$ coincides with 1 . This implies the series of Eq. (60) to converge for $\mu<1$. Further, the above-described spectrum of the matrix $\boldsymbol{R}$ may be used to derive the matrix $\boldsymbol{F}_{e}$ of Eq. (20). We obtain

$$
\boldsymbol{F}_{e}=\frac{1}{3(\mu+1)(\mu+2)}\left|\begin{array}{ccc}
3 \mu+5 & -1 & -1 \\
-1 & 3 \mu+5 & -1 \\
-1 & -1 & 3 \mu+5
\end{array}\right|
$$

It is seen that $\boldsymbol{F}_{\mathrm{e}}$ is a Hermitian (symmetric) matrix, eigenvalues of which coincide with $\left\{(\mu+2)^{-1}\right.$, $\left.(\mu+1)^{-1},(\mu+1)^{-1}\right\}$ and prove to be positive quantities for $\mu>0$. Thus, the positive-definite nature of our matrix $\boldsymbol{F}_{\mathrm{e}}$ also is beyond any doubt along with coincidence between $\boldsymbol{F}_{\mathrm{e}}$ and $\boldsymbol{R}$ for $\mu=0$.

Let us turn now to matrices of Eqs. (34)-(37). Using Eqs. (34) and (65), we obtain

$$
\boldsymbol{M}_{(1)}^{*}=\frac{\alpha}{108(\mu+1)^{2}(\mu+2)^{2}}\left|\begin{array}{ccc}
l(\mu) & -5 r(\mu) & -5 r(\mu) \\
-5 r(\mu) & r(\mu) & r(\mu) \\
-5 r(\mu) & r(\mu) & r(\mu)
\end{array}\right|(66)
$$


where

$$
l(\mu)=43 \mu^{2}+150 \mu+139, \quad r(\mu)=\mu^{2}+6 \mu+7 .
$$

Diagonal elements of the above matrix (i.e. $M_{(1) k k}^{*}$ ) are positive quantities for $\mu>0$ in accordance with Eq. (45). Thus, the increment $\boldsymbol{M}_{(1)}^{*}$ contributes to growth of CT abilities for attacks upon AOs $\chi_{1}^{*}, \chi_{2}^{*}$ and $\chi_{3}^{*}$ after introducing the nitrogen atom in accordance with our general conclusion. The matrix $\boldsymbol{N}_{(1)}^{*}$, in turn, easily results from Eqs. (35) and (65) and is as follows

$$
\boldsymbol{N}_{(\mathrm{l})}^{*}=-\frac{\alpha}{18(\mu+1)^{3}(\mu+2)^{3}}\left|\begin{array}{ccc}
t(\mu) & -s(\mu) & -s(\mu) \\
-s(\mu) & w(\mu) & w(\mu) \\
-s(\mu) & w(\mu) & w(\mu)
\end{array}\right|,
$$

where

$$
\begin{aligned}
& t(\mu)=2\left(3 \mu^{2}+10 \mu+9\right)(3 \mu+5), \\
& s(\mu)=9 \mu^{2}+29 \mu+24, \quad w(\mu)=2(2 \mu+3) .
\end{aligned}
$$

Diagonal elements of this matrix $\left(N_{(1) k k}^{*}\right)$ are negative quantities in accordance with Eq. (47). This conclusion may be entirely traced back to the minus sign of Eq. (35) and indicates the term $\boldsymbol{N}_{(1)}^{*}$ to contribute to lowering of CT abilities for attacks upon metapositioned carbon atoms after introducing the nitrogen atom.

For the remaining subset $\left\{\chi^{0}\right\}=\left\{\chi_{4}^{0}, \chi_{5}^{0}, \chi_{6}^{0}\right\}$, the relevant matrices $\boldsymbol{M}_{(1)}^{\mathrm{o}}$ and $\boldsymbol{N}_{(1)}^{\mathrm{o}}$ may be easily derived on the basis of Eqs. (42) and (44) after an additional invoking Eqs. (6), (62),(66) and (68). Let us confine ourselves here to diagonal elements of matrices concerned. These are

$$
\begin{aligned}
& M_{(1) 44}^{\mathrm{o}}=M_{(1) 66}^{\mathrm{o}}=-\frac{\alpha\left(17 \mu^{2}+54 \mu+47\right)}{12(\mu+1)^{2}(\mu+2)^{2}}, \\
& M_{(1) 55}^{\mathrm{o}}=-\frac{\alpha\left(11 \mu^{2}+54 \mu+59\right)}{12(\mu+1)^{2}(\mu+2)^{2}}, \\
& N_{(1) 44}^{\mathrm{o}}=N_{(1) 66}^{\mathrm{o}}=-\frac{\alpha\left(4 \mu^{3}+18 \mu^{2}+28 \mu+15\right)}{(\mu+1)^{3}(\mu+2)^{3}}, \\
& N_{(1) 55}^{\mathrm{o}}=-\frac{\alpha\left(\mu^{3}+9 \mu^{2}+25 \mu+21\right)}{(\mu+1)^{3}(\mu+2)^{3}}
\end{aligned}
$$

and prove to be negative quantities for $\mu>0$. This result also is in line with our general conclusions following from Eqs. (46) and (48).
Let us return to the subset $\left\{\chi^{*}\right\}$. To establish the actual sign of alteration in the CT ability for the attack upon the meta-positioned carbon atom when passing from benzene to pyridine, we need a sum of elements $\mathrm{M}_{(1) 22}^{*}$ and $\mathrm{N}_{(1) 22}^{*}$ (see Eq. (38)). Using Eqs. (66)-(69), we obtain

$$
\begin{aligned}
& D_{(1) 22}^{*}=M_{(1) 22}^{*}+N_{(1) 22}^{*}= \\
& =\frac{\alpha}{27(\mu+1)^{2}(\mu+2)^{2}}\left[\frac{\mu^{2}+6 \mu+7}{4}-\frac{3(2 \mu+3)}{(\mu+1)(\mu+2)}\right] .
\end{aligned}
$$

Analysis of this expression shows that the final sign of the total first order correction to the CT ability for the attack upon the AO $\chi_{2}^{*}\left(D_{(1) 22}^{*}\right)$ depends on the relative value of the principal parameter $\mu$ of the approaching electrophile in accordance with the expectation. Moreover, the above-mentioned correction takes a negative value for $0<\mu<0.685$. Hence, the total CT abilities for attacks upon the meta-positioned carbon atoms become reduced when passing from benzene to pyridine within a rather extended interval of $\mu$ values. This result along with negative signs of corrections $D_{(1) 44}^{\mathrm{o}}$ and $D_{(1) 55}^{\mathrm{o}}$ following from Eq. (70) illustrates the principal rule of our study about the suppressed charge transfer towards soft electrophiles in the case of heteroaromatic reactants.

Before finishing, let us compare absolute values of first order corrections to $\mathrm{CT}$ abilities for shifting positions of electrophile along the cycle, viz. $D_{(1) 44}^{*}$, $D_{(1) 22}^{\mathrm{o}}$ and $D_{(1) 55}^{\mathrm{o}}$ that are expected to describe relative reactivities of ortho-, meta- and para-positions of pyridine. As it turns out after application of Eqs. (66)-(70), absolute values of $D_{(1) 44}^{\circ}$ and $D_{(1) 55}^{\circ}$ exceed that of $D_{(1) 22}^{*}$ considerably. For example, we obtain that

$D_{(1) 22}^{*}=-0.025 \alpha, \quad D_{(1) 44}^{o}=-2.85 \alpha, \quad D_{(1) 55}^{o}=-3.85 \alpha$

for $\mu=0$ and

$D_{(1) 22}^{*}=-0.002 \alpha, \quad D_{(1) 44}^{\mathrm{o}}=-1.109 \alpha, \quad D_{(1) 55}^{\mathrm{o}}=-1.206 \alpha(73)$

for $\mu=0.5$, respectively. These results reflect the wellknown fact that exclusively meta-substitution is peculiar to pyridine. ${ }^{5,8}$ Thus, our approach acquires a certain advantage over the HOMO/LUMO concept. ${ }^{22-27}$

\section{CONCLUSIONS}

The above study gives algebraic expressions for matrices of $\mathrm{CT}$ abilities of systems participating in the $\mathrm{S}_{\mathrm{E}} 2$ reactions. Changes in the $\mathrm{CT}$ abilities due to 
introduction of the heteroatom into the reactant follow from diagonal elements of the above-mentioned matrices. In the case of a sufficiently soft electrophile, these elements are shown to be negative quantities for any derivative of an $\mathrm{AH}$ whatever the actual route of the reaction. Thus, the relevant rule of suppressed charge transfer between heteroaromatic compounds and electrophiles is proven. follows:

Significance of the results may be summarized as

1. The present study corroborates the empirical rule on the reduced relative reactivities in the $\mathrm{S}_{\mathrm{E}} 2$ reactions at all sites of heteroaromatic reactants $v s$. those of respective parent hydrocarbons.

2 . The results yield a common accounting for the above-mentioned empirical rule: The effect of suppression is shown to originate from predominance of the negative contributions to $\mathrm{CT}$ abilities over the alternating ones, the former being related to the increased distance in the energy scale between orbitals participating in the intermolecular charge transfer.

3. The above derivation provides us with conditions underlying the rule of suppressed CT abilities. Two principal requirements may be mentioned, viz. a sufficiently high electronaccepting ability of the reagent and absence of molecular orbitals described by one-electron energies of low absolute values in the one-electron spectrum of the reactant (substrate).

4. The results demonstrate importance of intermolecular interactions and insufficiency of reactivity descriptors based on an isolated reactant in the $\mathrm{S}_{\mathrm{E}} 2$ reactions.

5. The study allows us to reconcile an alternating pattern of excessive populations in the isolated heteroaromatic reactants and their suppressed reactivities towards electrophiles: The reactivity is shown to be representable more adequately by the respective CT ability, the final suppression of which, in turn, is compatible with an increased population.

6. The present study supplements the classical common rules on electronic structures of AHs and of their heteroatom-containing derivatives. The concept of common properties is now shown to refer also to responses of both AHs and their derivatives to external influences including that of an approaching reagent.

\section{REFERENCES}

1. F. A. Carroll, Perspectives on Structure and Mechanism in Organic Chemistry, Brooks/Cole, Pacific Grove, CA, 1998.

2. M. Edenborough, Organic Reaction Mechanisms. A Step by Step Approach, Taylor and Francies, London, 1999.
3. J. March, Advanced Organic Chemistry, Reactions, Mechanisms and Structure, Wiley/Interscience, New York, 1985.

4. F. A. Carey, Organic Chemistry, 2nd ed, McGraw-Hill, New York, 1992.

5. M. J. S. Dewar and R. C. Dougherty, The PMO Theory of Organic Chemistry, Plenum Press, New York, 1975.

6. V. I. Minkin, B. J. Simkin, and R. M. Mineev, Teorija Strojenija Molekul, Visshaja Shkola, Moscow, 1979 (in Russian).

7. A. R. Katritzky and C. D. Johnson, Angew. Chem. Intern. Ed. 6 (1967) 608-615

8. M. D'Auria, Tetrahedron Lett. 46 (2005) 6333-6336.

9. J. A. Joule and K. Mills, Heterocyclic Chem, 4th ed. Malden, MA: Blackwell Science, 2000.

10. J. M. Tedder and A. Nechvatal, Pictorial Orbital Theory, Pitman, London, 1985.

11. M. V. Basilevskii, Metod Molekuliarnych Orbit i Reaktsionnaya Sposobnost Organitcheskich Molekul, Khimia, Moscow, 1969 (in Russian)

12. R. Zahradnik and R. Polak, Elements of Quantum Chemistry, Plenum Press, New York, 1980.

13. N. D. Epiotis and S. Shaik, J. Am. Chem. Soc. 100 (1978) 29-33.

14. S. R. Gwaltney, S. V. Rosokha, M. Head-Gordon, and J. K. Kochi, J. Am. Chem. Soc. 125 (2003) 3273-3283.

15. A. N. Volkov, A. Y. Timoshkin, and A. V. Suvorov, Int. J. Quant. Chem. 104 (2005) 256-260.

16. P. M. Esteves, J. W. De M.Corneiro, S. P. Cardoso, A. G. H. Barbosa, K. K. Laali, G. Rasul, G. K. Surya Prakash, and G. A. Olah, J. Am. Chem. Soc. 125 (2003) 4836-4849.

17. L. I. Belenkii, I. D. Nesterov, and N. D. Chuvylkin, Chem. Heterocyclic Comp. 44 (2008) 1339-1346.

18. E. E. Stashenko, J. R. Martinez, G. Tafurt-Garcia, A. Palma, and J. M. Bofill, Tetrahedron 64 (2008) 7407-7418.

19. M. Eckert-Maksic, M. Klessinger, D. Kovacek, and Z. B. Maksic, J. Phys. Org. Chem. 9 (1998) 269-278.

20. A. V. Sokolov, Int. J. Quant. Chem. 100 (2004) 1-12.

21. G. Raos, J. Gerratt, P. B. Karadakov, D. L. Cooper, and M. Raimondi, J. Chem. Soc. Faraday Trans. 91 (1995) 4011-4030.

22. K. Fukui and H. Fujimoto, Bull. Chem. Soc. Japan 41 (1968) 1989-1997; 42 (1969) 3399-3409.

23. H. Fujimoto, S. Yamabe, and K. Fukui, Bull. Chem. Soc. Japan 44 (1971) 2936-2941.

24. K. Fukui, Angew. Chem. Int. Ed. 21 (1982) 801-809.

25. G. Klopman and R. F. Hudson, Theor. Chim. Acta 8 (1967) 165-172.

26. G. Klopman, J. Am. Chem. Soc. 90 (1968) 223-234.

27. K. Fukui, Acc. Chem. Res. 4 (1971) 57-64

28. K. Fukui, Science 218 (1982) 747-754.

29. G. Klopman, (Ed.), Chemical Reactivity and Reaction Paths, John Wiley and Sons, Inc., New York, London, Sydney, Toronto, 1974.

30. I. Fleming, Frontier Orbitals and Organic Chemical Reactions, Wiley, Chichester, 1976.

31. H. Hirao and T. Ohwada, J. Phys. Chem A 107 (2003) 2875-2881.

32. Z. Zhou and R. G. Parr, J. Am. Chem. Soc. 112 (1990) 5720-5724.

33. A. Ciesielski, T. M. Krygowsky, M. K. Cyranski, M. A. Dobrowolski, and A. T. Balaban, J. Chem. Inf. Model. 49 (2009) 369-376.

34. M. G. Bures, B. L. Roos-Kozel, and W. L. Jorgensen, J. Org. Chem. 50 (1985) 4490-4498.

35. J. S. M. Anderson, J. Melin, and P. W. Ayers, J. Chem. Theory Comput. 3 (2007) 375-389.

36. P. Perez, L. R. Domingo, M. Duque-Norena, and E. Chamorro, J. Mol. Struct. (Theochem) 895 (2009) 86-91.

37. A. M. Vos, R. A. Schoonheydt, F. De Proft, and P. Geerlings, $J$. Catalysis 220 (2003) 333-346.

38. L. Meneses, W. Tiznado, R. Contreras, and P. Fuentalba, Chem. 
Phys. Lett. 383 (2004) 181-187.

39. P. Geerlings and F. De Proft, Int. J. Mol. Sci. 3 (2002) 276-309.

40. R. G. Parr and W. Yang, J. Am. Chem. Soc. 106 (1984) 4049-4050.

41. W. Yang, R. G. Parr, and R. Pucci, J. Chem. Phys. 81 (1984) 2862-2863.

42. P. W. Ayers and M. Levy, Theor. Chem. Acc. 103 (2000) 353-360.

43. P. W. Ayers, J. S. M. Anderson, and L. J. Bartolotti, Int. J. Quant. Chem. 101 (2005) 520-534.

44. E. Chamorro and P. Perez, J. Chem. Phys. 123 (2005) 114107-10.

45. C. A. Coulson and H. C. Longuet-Higgins, Proc. Roy. Soc. London Ser. A 192 (1947) 16-32.

46. I. Gutman, Z. Naturforsch. A 36 (1981) 1112-1114.

47. V. Gineityte, Int. J. Quant. Chem. 105 (2005) 232-245.

48. G. G. Hall, Proc. Roy. Soc. London Ser. A 229 (1955) 251-259.

49. N. Trinajstic in: G.A.Segal (Ed.), Semiempirical Methods of Electronic Structure Calculations, Part A, Techniques, Plenum Press, New York and London, 1977.

50. D. M. Cvetkovic, M. Doob, and H. Sachs, Spectra of Graphs. Theory and Application, VEB Deutscher Verlag der Wissenschaften, Berlin, 1980.

51. N. Trinajstic, Chemical Graph Theory, 1st ed., Parts I and II, CRC Press, Boca Raton, FL, 1983.

52. V. P. Feshin and M. Yu. Konshin, Russ. Chem. Bull. 45 (1996) 2501-2504.

53. V. P. Feshin, E. V. Feshina, and L. I. Zhizhina, Chem. Heterocyclic Comp. 42 (2006) 1435-1443.

54. G. Kollenz, S. Holzer, C. O.Kappe, T. S. Dalvi, W. M. F. Fabian,
H. Sterk, Ming Wah Wong, and C. Wentrup, Eur. J. Org. Chem. 2001 (2001) 1315-1322.

55. J. Jie-Jack Li (Ed.), Name Reactions in Heterocyclic Chemistry Wiley, Hoboken, NJ, 2005.

56. D. E. Lushnikov and G. Sello, J. Chem. Inf. Comput. Sci. 35 (1995) 1060-1067.

57. Tse-Lok Ho, Res. Chem. Intermediat. 9 (1988) 117-140.

58. E. V. Babaev, J. Russ. Mendeleev Chem. Soc. 38 (1994) 54-65.

59. V. Gineityte, Int. J. Quant. Chem. 101 (2005) 274-282.

60. V. Gineityte, Int. J. Quant. Chem. 68 (1998) 119-127.

61. V. Gineityte, Int. J. Quant. Chem. 72 (1999) 559-570.

62. V. Gineityte, Lithuanian J. Phys. 44 (2004) 219-236.

63. V. Gineityte, Int. J. Quant. Chem. 106 (2006) 2145-2160.

64. V. Gineityte, Croat. Chem. Acta 81 (2008) 487-499.

65. V. Gineityte, J. Mol. Struct. (Theochem) 343 (1995) 183-194.

66. V. Gineityte, J. Mol. Struct. (Theochem) 546 (2001) 107-117.

67. V. Gineityte, J. Mol. Struct. (Theochem) $\mathbf{5 8 5}$ (2002) 15-25.

68. V. Gineityte, Z. Naturforsch, 64A (2008) 132-148.

69. M. M. Mestetchkin, The Density Matrix Method in Quantum Chemistry, Naukova Dumka, Kiev, 1977 (in Russian).

70. V. Gineityte, Int. J. Quant. Chem. 108 (2008) 1141-1154.

71. V. Gineityte, Int. J. Quant. Chem. 94 (2003) 302-316.

72. R. A. Horn and C. R. Johnson, Matrix Analysis, Cambridge Univ. Press, Cambridge, 1986.

73. P. Lankaster, Theory of Matrices, Academic Press, New York, London, 1969.

74. R. Bellman, Introduction to Matrix Analysis, McGraw-Hill, New York, 1960.

75. V. F. Traven, Electronic Structure and Properties of Organic Compounds, Khimia, Moscow, 1989 (in Russian). 